\title{
THE SIMPLY LAMINATED MICROSTRUCTURE IN MARTENSITIC CRYSTALS THAT UNDERGO A CUBIC TO ORTHORHOMBIC PHASE TRANSFORMATION
}

\author{
KAUSHIK BHATTACHARYA, BO LI, AND MITCHELL LUSKIN
}

\begin{abstract}
We study simply laminated microstructures of a martensitic crystal capable of undergoing a cubic to orthorhombic transformation of type $\mathcal{P}^{(432)} \rightarrow \mathcal{P}^{(222)^{\prime}}$. The free energy density modeling such a crystal is minimized on six energy wells that are pairwise rank-one connected. We consider the energy minimization problem with Dirichlet boundary data compatible with an arbitrary but fixed simple laminate. We first show that for all but a few isolated values of transformation strains, this problem has a unique Young measure solution solely characterized by the boundary data that represents the simply laminated microstructure. We then present a theory of stability for such a microstructure, and apply it to the conforming finite element approximation to obtain the corresponding error estimates for the finite element energy minimizers.
\end{abstract}

\section{INTRODUCTION}

One of the most frequently observed microstructures of a martensitic crystal is a fine-scale twin or a simple laminate which is an array of parallel layers with extremely fine layer thickness. Such a crystal undergoes a martensitic phase transformation - a diffusionless, structural, reversible phase transformation - from a high-temperature, high-symmetry austenite phase to a low-temperature, low-symmetry martensite phase. The change in symmetry gives rise to multiple variants of martensite - lattices that are identical in structure but oriented differently with respect to the parent austenite lattice. Below the transformation temperature, the variants of martensite often arrange themselves to form a microstructure - a fine-scale mixture of the different variants.

A recently developed geometrically nonlinear theory predicts the martensitic microstructure by energy minimization, see for example $[9,10,11,13,15,20,21,24,27,34]$

Date: April 22, 2003.

1991 Mathematics Subject Classification. 49J45, 65N15, 65N30, 73C50, 73G05, 73K20, 73V05.

Key words and phrases. martensitic crystals, cubic to orthorhombic transformation, simply laminated microstructure, volume fractions, the six-well problem, Young measure, stability, finite element approximation, error estimates.

This paper was completed while Kaushik Bhattacharya was visiting the Max-Planck Institute for Mathematics in the Sciences in Leipzig. He was grateful for the hospitality of the institute. He was also partially supported by the NSF through grant CMS-9457573 and by the AFOSR through grant F49620-95-1-0109. Bo Li was partially supported by the DARPA/URI/ONR through grant N0001492-J-1890 and by the NSF through grant DMS 96-15854. Mitchell Luskin was partially supported by the NSF through grant DMS 95-05077, by the AFOSR through grant AF/F 49620-97-1-0187, by the ARO through grant DAAG55-98-1-0335, and by a grant from the Minnesota Supercomputer Institute. 
and the references therein. In this theory, the free energy density of the crystal below the transformation temperature is minimized on several energy wells representing the martensitic variants. The total energy fails therefore to be weakly lower semicontinuous and energy-minimizing sequences may develop fine-scale oscillations and converge only in some weak sense. These fine-scale oscillations manifest themselves as the microstructure, and are described mathematically by the notion of Young measure $[6,10,26,41,43]$. Thus, the energy minimization problem may not have classical solutions but only Young measure solutions. Further, it is atypical to expect the uniqueness of the Young measure solutions and consequently it is difficult to obtain error estimates for finite element minimizers.

A fine-scale twin or a simple laminate is a microstructure consisting of alternating bands of two different variants, and the corresponding Young measure is a convex combination of two Dirac masses. One can obtain such a microstructure as an energyminimizing deformation if and only if two variants satisfy an algebraic restriction of compatibility, that is, they are "rank-one connected" or "twin-related".

In this paper, we are concerned with the simply laminated microstructure of a martensitic crystal that can undergo a cubic to orthorhombic transformation of type $\mathcal{P}^{(432)} \rightarrow \mathcal{P}^{(222)^{\prime} 1}$. A typical example of such a crystal is the shape-memory alloy Copper-Aluminum-Nickel ( $\mathrm{Cu}-\mathrm{Al}-\mathrm{Ni}$ ) single crystal which is widely used for experimental studies of the shape-memory effect, see for example [2, 16, 22, 23, 38, 42], and which has motivated various theoretical studies including [2, 11, 28, 29]. A crystal capable of undergoing such a transformation has six variants of martensite, each pair of variants being rank-one connected. All the rank-one connections can be classified into compound twins and noncompound (type I or type II) twins $[10,11,16,38]$. Thus, the crystal is capable of a wide variety of simple laminates, and we show in this paper that these microstructures are special in a certain sense.

We study the energy minimization problem with a Dirichlet boundary condition compatible with an arbitrary but fixed simple laminate - a convex combination of two compatible energy-minimizing states. Our analysis shows that the deformation gradient for this problem takes values essentially in only two of the six available wells. This gives some rigorous justification for the assumption made in most of analytical and computational investigations of the Chu-James experiment (cf. [16]) that the deformation gradient can be modeled by a two-well problem [1, 2, 7, 25, 28, 29].

Our main results are as follows. First, we use the "minors relations" to show that for all but a few isolated values of transformation strains, the energy minimization problem has a unique Young measure solution solely characterized by the boundary data that represents the simply laminated microstructure. This is true for both compound and noncompound twins. Second, we develop a theory of stability for such a microstructure. This theory consists of a series of estimates via elastic energies for the reduction of the six-well problem to a two-well problem, for the approximation in strong topology of the

\footnotetext{
${ }^{1}$ There are two types of cubic to orthorhombic martensitic transformations. We are unaware of any material that can undergo a transformation of the other type $\mathcal{P}^{(432)} \rightarrow \mathcal{P}^{(222)}$, and hence we do not consider it.
} 
directional derivative of deformations in any direction tangential to the "twin planes", for the approximation of volume fractions of deformation gradients participating in the microstructure, and for the approximation of nonlinear integrals of deformation gradients. Finally, we apply the theory of stability to the conforming finite element approximation of the simply laminated microstructure to obtain corresponding error estimates for finite element energy minimizers.

Similar results are known for the orthorhombic to monoclinic (two-well) and the cubic to tetragonal (three-well) transformations. Ball and James [9, 10] proved the uniqueness of the Young measure for these transformations. Luskin [33] gave a stability theory for microstructure and used this theory to analyze the orthorhombic to monoclinic transformation. Li and Luskin extended this theory to the cubic to tetragonal transformation [28, 30] and to laminates with varying volume fractions [32]. The fact that the energy density for the cubic to orthorhombic transformation has six wells makes this transformation significantly more difficult to analyze than the two and three-well transformations since the additional wells give the crystal more freedom to deform without the cost of additional energy. The numerical analysis of both conforming and nonconforming finite element approximations for the two-well and three-well transformations has been presented by $\mathrm{Li}$ and Luskin $[30,31,32,33]$. The stability theory was also used by Luskin and Ma to analyze the microstructure in ferromagnetic crystals [35].

We organize the rest of this paper as follows. In $\S 2$ we describe the energy minimization problem for the crystal in discussion. In $\S 3$ we prove the uniqueness of the Young measure for the energy minimization problem with a boundary data consistent with a simply laminated microstructure. In $\S 4$ we present the theory of stability for a simply laminated microstructure. Finally, in $\S 5$ we apply the theory of stability to obtain corresponding error estimates for finite element quasi-minimizers.

\section{The Energy Minimization Problem}

We denote by $\Omega$ the reference configuration of the crystal in discussion which is taken to be the homogeneous cubic state at the transformation temperature. We assume that $\Omega \subset \mathbb{R}^{3}$ is a bounded domain with a Lipschitz continuous boundary, $\partial \Omega$. We also denote a deformation of the crystal by $y: \Omega \rightarrow \mathbb{R}^{3}$ and its gradient by $\nabla y: \Omega \rightarrow \mathbb{R}^{3 \times 3}$, where $\mathbb{R}^{3 \times 3}$ is the set of all $3 \times 3$ real matrices. We seek to minimize the total energy

$$
\mathcal{E}(y):=\int_{\Omega} \phi(\nabla y(x)) d x
$$

over an admissible class $\mathcal{A}$ of deformations, where $\phi: \mathbb{R}^{3 \times 3} \rightarrow \mathbb{R}$ is the free energy density per unit volume of the reference configuration of the crystal at a fixed temperature below the transformation temperature.

We assume that the free energy density is rotationally invariant:

$$
\phi(R F)=\phi(F) \quad \forall F \in \mathbb{R}^{3 \times 3}, \forall R \in \mathrm{SO}(3),
$$

where $\mathrm{SO}(3)$ is the set of all proper rotations. To model the cubic to orthorhombic transformation of type $\mathcal{P}^{(432)} \rightarrow \mathcal{P}^{(222)^{\prime}}$, we also assume that the free energy density is 
minimized on the six energy wells

$$
\mathcal{U}_{i}:=\operatorname{SO}(3) U_{i}=\left\{R U_{i}: R \in \mathrm{SO}(3)\right\}, \quad i=1, \cdots, 6,
$$

where $U_{1}, \cdots, U_{6}$ are the transformation matrices with respect to the cubic basis $\left\{e_{1}, e_{2}, e_{3}\right\}$ of the six variants of martensite, respectively. So, by adding a constant, we may and do assume that

$$
\begin{array}{ccl}
\phi(F) \geq 0 \quad & \forall F \in \mathbb{R}^{3 \times 3} \\
\phi(F)=0 \quad \text { if and only if } \quad F \in \mathcal{U}:=\mathcal{U}_{1} \cup \cdots \cup \mathcal{U}_{6} .
\end{array}
$$

Finally, we assume that the free energy density $\phi: \mathbb{R}^{3 \times 3} \rightarrow \mathbb{R}$ is continuous and satisfies certain growth conditions to be made precise in the next sections.

We recall that the six transformation matrices are given by $[10,11,16]$

$$
\begin{aligned}
U_{1} & =\left(\begin{array}{lll}
\zeta & 0 & 0 \\
0 & \xi & \eta \\
0 & \eta & \xi
\end{array}\right), & U_{2} & =\left(\begin{array}{ccc}
\zeta & 0 & 0 \\
0 & \xi & -\eta \\
0 & -\eta & \xi
\end{array}\right), \\
U_{3} & =\left(\begin{array}{lll}
\xi & 0 & \eta \\
0 & \zeta & 0 \\
\eta & 0 & \xi
\end{array}\right), & U_{4} & =\left(\begin{array}{ccc}
\xi & 0 & -\eta \\
0 & \zeta & 0 \\
-\eta & 0 & \xi
\end{array}\right), \\
U_{5} & =\left(\begin{array}{lll}
\xi & \eta & 0 \\
\eta & \xi & 0 \\
0 & 0 & \zeta
\end{array}\right), & U_{6} & =\left(\begin{array}{ccc}
\xi & -\eta & 0 \\
-\eta & \xi & 0 \\
0 & 0 & \zeta
\end{array}\right) .
\end{aligned}
$$

The material parameters $\xi, \eta$, and $\zeta$ are defined by

$$
\xi=\frac{\alpha+\gamma}{2}, \quad \eta=\frac{\alpha-\gamma}{2}, \quad \zeta=\beta,
$$

where $\alpha, \beta$, and $\gamma$ are the transformation strains, that is, the principal strains of a linear transformation that transforms the cubic lattice to the orthorhombic lattice. For the shape-memory alloy Cu-14.2 at.\% Al-4.3 at.\% Ni single crystal at room temperature, the experimental values of these transformation strains are [38]

$$
\alpha=1.0691, \quad \beta=0.9178, \quad \gamma=1.0231 \text {. }
$$

Throughout this paper, we shall assume without loss of generality that

$$
\eta>0, \quad \xi>\eta, \quad \zeta>0 .
$$

We can assume the first one by renumbering the variants if necessary and the last two by the requirement that a transformation matrix is always symmetric positive definite.

The transformation matrices of the variants are symmetry-related. These symmetry relations are summarized in the following lemma that can be easily proved by verification, cf. [10, 12, 16].

Lemma 2.1. For any indices $i, j \in\{1, \cdots, 6\}$ with $i \neq j$, there exists a rotation $R_{i j}$ of $180^{\circ}$ about some $e_{i j} \in \mathbb{R}^{3}, R_{i j}=R\left[180^{\circ}, e_{i j}\right]$, such that

$$
R_{i j}^{T} U_{i} R_{i j}=U_{j}
$$


Moreover, up to scalar multiples,

$$
\begin{gathered}
e_{i j} \in\left\{ \pm e_{1}, \pm e_{2}, \pm e_{3}\right\}, \\
\text { if }(i, j) \in\{(1,2),(2,1),(3,4),(4,3),(5,6),(6,5)\}, \text { and } \\
e_{i j} \in\left\{e_{k} \pm e_{l}: k, l=1,2,3, k \neq l\right\},
\end{gathered}
$$

otherwise.

We say that the energy wells $\mathcal{U}_{i}$ and $\mathcal{U}_{j}$ are rank-one connected if there exists $Q \in$ $\mathrm{SO}(3)$ such that the difference $Q U_{i}-U_{j}$ is a rank-one matrix, that is, if

$$
Q U_{i}-U_{j}=a \otimes n
$$

for some $a, n \in \mathbb{R}^{3}$ with $a, n \neq 0$. Here, for any $v, w \in \mathbb{R}^{3}, v \otimes w$ is the tensor product defined by $(v \otimes w) u=(w \cdot u) v$ for $u \in \mathbb{R}^{3}$, or equivalently, $v \otimes w \in \mathbb{R}^{3 \times 3}$ is the matrix with entries $(v \otimes w)_{k l}=v_{k} w_{l}$ with respect to the orthonormal basis $\left\{e_{1}, e_{2}, e_{3}\right\}$. The equation (2.7) is known as the "twinning equation". According to the classical Hadamard compatibility condition, there exists a continuous deformation $y$ whose gradient takes the value $Q_{1} U_{i}$ on one side of a given plane and $Q_{2} U_{j}$ on the other side for some $Q_{1}, Q_{2} \in \mathrm{SO}(3)$ if and only if $Q_{1} U_{i}-Q_{2} U_{j}$ is a matrix of rank-one, that is, if and only if the energy wells $\mathcal{U}_{i}$ and $\mathcal{U}_{j}$ are rank-one connected as in (2.7). Further, the gradient can jump only on planes with normal $n$. Such a deformation describes a twin with $n$ the normal of the twinning plane.

The following lemma summarizes all the rank-one connections between the six wells. See $[9,12,16]$ for a proof.

Lemma 2.2. (1) For each $i \in\{1, \cdots, 6\}$, the energy well $\mathcal{U}_{i}$ is not rank-one connected to itself.

(2) For any $i, j \in\{1, \cdots, 6\}$ with $i \neq j$, there are exactly two rank-one connections between the energy wells $\mathcal{U}_{i}$ and $\mathcal{U}_{j}$, that is, there are exactly two distinct rotations $Q_{i j} \in S O(3)$ such that $Q_{i j} U_{i}-U_{j}$ are rank-one matrices. Moreover, the solutions $(a, n) \in \mathbb{R}^{3} \times \mathbb{R}^{3}$ to the twinning equation (2.7) are given by

$$
a=a_{1}=\frac{2}{\rho}\left(\frac{U_{j}^{-1} e}{\left|U_{j}^{-1} e\right|^{2}}-U_{j} e\right), \quad n=n_{1}=\rho e,
$$

and

$$
a=a_{2}=\rho U_{j} e, \quad n=n_{2}=\frac{2}{\rho}\left(e-\frac{U_{j}^{2} e}{\left|U_{j} e\right|^{2}}\right),
$$

respectively, where $e=e_{i j} /\left|e_{i j}\right|$ is the unit vector of the axis of the rotation $R_{i j}=$ $R\left[180^{\circ}, e_{i j}\right]$ given in Lemma 2.1, and $\rho \in \mathbb{R}$ is a nonzero constant. We can find the corresponding rotation $Q$ by substituting back into (2.7).

If $(i, j) \in\{(1,2),(2,1),(3,4),(4,3),(5,6),(6,5)\}$, the two normals $n_{1}$ and $n_{2}$ are both axes of rotations of $180^{\circ}$ that lead to the symmetry relation (2.6). In this case, we say that (2.7) determines a compound twin. Otherwise, only $n_{1}=e$ is the axis of 
a rotation of $180^{\circ}$ that leads to the symmetry relation (2.6). In this case, we say that (2.7) determines a noncompound twin and the solution (2.8) gives a type I twin and (2.9) a type II twin.

We identify microstructures with minimizing sequences of the energy functional $\mathcal{E}$ defined in (2.1). Of particular interest here is a simple laminate. Thus, we assume throughout the rest of the paper that $i, j \in\{1, \cdots, 6\}$ with $i \neq j$, and that $Q \in \operatorname{SO}(3)$ and $a, n \in \mathbb{R}^{3}$ with $|n|=1$ satisfy the twinning equation (2.7). For an arbitrary but fixed $\lambda \in(0,1)$, we also denote

$$
F_{\lambda}:=\lambda Q U_{i}+(1-\lambda) U_{j}=U_{j}+\lambda a \otimes n .
$$

Now, for each integer $k \geq 1$, we can construct a deformation $\tilde{y}_{k} \in W^{1, \infty}\left(\Omega ; \mathbb{R}^{3}\right)$ such that its gradient $\nabla \tilde{y}_{k}$ takes the value $Q U_{i}$ and $U_{j}$ alternatively in parallel layers with thickness $\lambda / k$ and $(1-\lambda) / k$ respectively and with normal $n$. It follows from (2.1) and (2.3) that $\mathcal{E}\left(\tilde{y}_{k}\right)=0$. Moreover, $\tilde{y}_{k} \stackrel{*}{\rightarrow} \tilde{y}$ in $W^{1, \infty}\left(\Omega ; \mathbb{R}^{3}\right)$ as $k \rightarrow \infty$, where $\nabla \tilde{y}=F_{\lambda}$. With a slight modification of $\tilde{y}_{k}$ on a set near the boundary whose measure goes to zero, we obtain a sequence $\left\{y_{k}\right\} \subset W^{1, \infty}\left(\Omega ; \mathbb{R}^{3}\right)$ such that $y_{k}(x)=F_{\lambda} x$ for $x \in \partial \Omega$, $y_{k} \stackrel{*}{\rightarrow} F_{\lambda} x$ in $W^{1, \infty}\left(\Omega ; \mathbb{R}^{3}\right)$, and

$$
\lim _{k \rightarrow \infty} \mathcal{E}\left(y_{k}\right)=0,
$$

cf. $[9,15,34]$. We say that this sequence generates a simple laminate with normal $n$ composed of the gradients $Q U_{i}$ and $U_{j}$. Notice that for the limiting deformation $z(x):=F_{\lambda} x, x \in \Omega$, we have $\mathcal{E}(z)>0$ by (2.3) and the following lemma.

Lemma 2.3. We have for any $\lambda \in(0,1)$ that $F_{\lambda} \notin \mathcal{U}$.

Proof. Obviously, $F_{\lambda} \in \mathcal{U}$ is equivalent to $F_{\lambda}^{T} F_{\lambda}=U_{k}^{2}$ for some $k \in\{1, \cdots, 6\}$. This, together with (2.10), implies that

$$
U_{j}^{2}+\lambda n \otimes U_{j} a+\lambda U_{j} a \otimes n+\lambda^{2}|a|^{2} n \otimes n=U_{k}^{2} .
$$

Calculating the trace of relevant terms and noticing the fact that the trace of $U_{j}^{2}$ is the same as that of $U_{k}^{2}$, we obtain

$$
2 \lambda n \cdot U_{j} a+\lambda^{2}|a|^{2}|n|^{2}=0 .
$$

But the solutions of this equation are $\lambda=0$ and $\lambda=1$, if $a$ and $n$ are given by (2.8) or (2.9). Hence, $F_{\lambda} \notin \mathcal{U}$ if $\lambda \in(0,1)$.

In the rest of the paper, we will confine ourselves to deformations which satisfy the boundary condition compatible with a simple laminate:

$$
y(x)=F_{\lambda} x, \quad x \in \partial \Omega .
$$

We thus assume that the set of admissible deformations $\mathcal{A}$ satisfies

$$
W_{\lambda}^{1, \infty}\left(\Omega ; \mathbb{R}^{3}\right) \subseteq \mathcal{A} \subseteq W_{\lambda}^{1,1}\left(\Omega ; \mathbb{R}^{3}\right)
$$

where for $1 \leq p \leq \infty$ we denote

$$
W_{\lambda}^{1, p}\left(\Omega ; \mathbb{R}^{3}\right):=\left\{y \in W^{1, p}\left(\Omega ; \mathbb{R}^{3}\right): y(x)=F_{\lambda} x \text { for } x \in \partial \Omega\right\},
$$


and where the boundary value is understood in the sense of trace. The simple laminate construction proves the following lemma.

Lemma 2.4. Suppose the set of admissible deformations $\mathcal{A}$ satisfies (2.13). Then

$$
\inf _{y \in \mathcal{A}} \mathcal{E}(y)=0 .
$$

\section{The Uniqueness of the Young Measure}

In this section, we assume that the continuous energy density $\phi: \mathbb{R}^{3 \times 3} \rightarrow \mathbb{R}$ also satisfies the following growth condition

$$
\phi(F) \geq c_{0}+c_{1}\|F\|^{p} \quad \forall F \in \mathbb{R}^{3 \times 3},
$$

for some real constants $c_{0}, c_{1}$, and $p$ such that $c_{1}>0$ and $p>1$, where $\|F\|$ is the matrix norm of $F=\left(F_{k l}\right) \in \mathbb{R}^{3 \times 3}$ defined by

$$
\|F\|^{2}:=\operatorname{trace}\left(F^{T} F\right)=\sum_{k, l=1}^{3} F_{k l}^{2} .
$$

Correspondingly, we define the set of admissible deformations to be

$$
\mathcal{A}:=W_{\lambda}^{1, p}\left(\Omega ; \mathbb{R}^{3}\right):=\left\{y \in W^{1, p}\left(\Omega ; \mathbb{R}^{3}\right): y(x)=F_{\lambda} x \text { for } x \in \partial \Omega\right\} .
$$

Let $\left\{y_{k}\right\}$ be a sequence of deformations bounded in $W^{1, p}\left(\Omega ; \mathbb{R}^{3}\right)$. Following $[6,10,43]$, we recall that the Young measure associated with a subsequence of $\left\{\nabla y_{k}\right\}$, still denoted by $\left\{\nabla y_{k}\right\}$, is a family of probability measures $\left\{\nu_{x}: x \in \Omega\right\}$ on the gradient space $\mathbb{R}^{3 \times 3}$ such that for any continuous function $f: \mathbb{R}^{3 \times 3} \rightarrow \mathbb{R}$ with a suitable growth rate at infinity and any measurable subset $\omega \subseteq \Omega$,

$$
f\left(\nabla y_{k}\right) \rightarrow \bar{f} \quad \text { in } L^{1}(\omega)
$$

where

$$
\bar{f}(x):=\int_{\mathbb{R}^{3 \times 3}} f(F) d \nu_{x}(F), \quad x \in \Omega .
$$

We now present the main result of this section - the uniqueness of the Young measure. We shall denote by $\delta_{G}$ the Dirac measure on $\mathbb{R}^{3 \times 3}$ centered at $G \in \mathbb{R}^{3 \times 3}$.

Theorem 3.1. Let $\phi: \mathbb{R}^{3 \times 3} \rightarrow \mathbb{R}$ satisfy (2.3) and (3.1) with $p>1$, and $\mathcal{A}$ be defined by (3.2). Let $\left\{y_{k}\right\} \subset \mathcal{A}$ be an energy-minimizing sequence defined by (2.11) and $\left\{\nu_{x}: x \in \Omega\right\}$ the Young measure associated with a subsequence of $\left\{\nabla y_{k}\right\}$.

(1) Suppose the rank-one connection (2.7) used in the definition of $F_{\lambda}$ determines a compound twin. Then the Young measure is unique and

$$
\nu_{x}=\lambda \delta_{Q U_{i}}+(1-\lambda) \delta_{U_{j}} \quad \text { a.e. } x \in \Omega
$$

for all the parameters $\xi, \eta$, and $\zeta$ satisfying (2.5), except those such that

$$
\zeta^{2}=\xi^{2}+\eta^{2} \quad \text { or } \quad \zeta^{2}=\frac{\left(\xi^{2}-\eta^{2}\right)^{2}}{\xi^{2}+\eta^{2}}
$$

in which case the Young measure is not unique if $\lambda=1 / 2$. 
(2) Suppose the rank-one connection (2.7) used in the definition of $F_{\lambda}$ determines a noncompound twin. If it is a type I twin, then the Young measure is unique and (3.3) holds true for all the parameters $\xi, \eta$, and $\zeta$ satisfying (2.5), except those such that

$$
\zeta=\xi+\eta
$$

in which case the Young measure is not unique if $\lambda=\frac{1}{3}\left(4-\frac{\eta}{\xi}-\frac{\xi}{\eta}\right) \in(0,1)$.

If it is a type II twin, then the Young measure is unique and (3.3) holds true for all the parameters $\xi, \eta$, and $\zeta$ satisfying (2.5), except those such that

$$
\zeta=\xi-\eta
$$

in which case the Young measure is not unique if $\lambda=\frac{1}{3}\left(4-\frac{\eta}{\xi}-\frac{\xi}{\eta}\right) \in(0,1)$.

As a direct consequence of the theorem, we have the following result on the nonexistence of energy minimizers.

Corollary 3.1. If the parameters $\xi, \eta$, and $\zeta$ satisfying (2.5) lead to the unique Young measure (3.3) and $\lambda \in(0,1)$, then there does not exist any $y \in \mathcal{A}$ such that

$$
\mathcal{E}(y)=\min _{z \in \mathcal{A}} \mathcal{E}(z)
$$

Proof. If there existed $y \in \mathcal{A}$ satisfying (3.7), then $\left\{y_{k}\right\} \subset \mathcal{A}$ with each $y_{k}:=y$ would be an energy-minimizing sequence. Any Young measure associated with the corresponding sequence of gradients would be given by $\nu_{x}=\delta_{\nabla y(x)}$ for almost every $x \in \Omega$, which obviously contradicts (3.3).

We shall frequently use the following well-known result that a subdeterminant of the gradient is a null-Lagrangian $[4,5,8,18,19]$.

Lemma 3.1. For any $y \in W^{1,2}\left(\Omega ; \mathbb{R}^{3}\right)$ that satisfies the boundary condition (2.12), we have

$$
\begin{aligned}
\int_{\Omega} \nabla y(x) d x & =\int_{\Omega} F_{\lambda} d x, \\
\int_{\Omega} \operatorname{Cof} \nabla y(x) d x & =\int_{\Omega} \operatorname{Cof} F_{\lambda} d x .
\end{aligned}
$$

Proof of Theorem 3.1. It is easy to see from the growth condition (3.1) that there exists a subsequence of $\left\{y_{k}\right\}$, not relabeled, such that

$$
y_{k} \rightarrow y \quad \text { in } W^{1, p}\left(\Omega ; \mathbb{R}^{3}\right)
$$

for some $y \in \mathcal{A}$. Moreover (cf. [10]),

$$
\operatorname{supp} \nu_{x} \subseteq \mathcal{U}=\mathcal{U}_{1} \cup \cdots \cup \mathcal{U}_{6} \quad \text { a.e. } x \in \Omega \text {. }
$$

By Zhang's theorem (cf. [45], also [37]), we may assume that the family of Young measures are in fact generated by a $W^{1, \infty}\left(\Omega ; \mathbb{R}^{3}\right)$ bounded sequence of deformations which satisfy the boundary condition (2.12). Moreover, this sequence converges weak-* 
in $W^{1, \infty}\left(\Omega ; \mathbb{R}^{3}\right)$ to the same $y \in \mathcal{A}$ (hence $y \in W^{1, \infty}\left(\Omega ; \mathbb{R}^{3}\right)$ ). We therefore have the following part of the "minors relations" $[4,5,10,19,36,40]$

$$
\begin{gathered}
\nabla y(x)=\int_{\mathbb{R}^{3 \times 3}} F d \nu_{x}(F) \quad \text { a.e. } x \in \Omega, \\
\operatorname{Cof} \nabla y(x)=\int_{\mathbb{R}^{3 \times 3}} \operatorname{Cof} F d \nu_{x}(F) \quad \text { a.e. } x \in \Omega .
\end{gathered}
$$

For each $k \in\{1, \cdots, 6\}$, we now denote

$$
\tau_{k}:=\frac{1}{\text { meas } \Omega} \int_{\Omega} \nu_{x}\left(\mathcal{U}_{k}\right) d x \geq 0
$$

It follows from (3.10) that

$$
\sum_{k=1}^{6} \tau_{k}=1
$$

By the result of Ball and James [10] on the two-well problem, we need only to show that $\tau_{k}=0$ for any $k \in\{1, \cdots, 6\}$ such that $k \neq i$ and $k \neq j$.

By (3.10), (3.8) and (3.11), we have for any $w \in \mathbb{R}^{3}$ that

$$
\begin{aligned}
\rho_{1}(w) & :=\sum_{k=1}^{6} \tau_{k}\left(\left|U_{k} w\right|^{2}-\left|F_{\lambda} w\right|^{2}\right) \\
& =\frac{1}{\operatorname{meas} \Omega} \int_{\Omega} \int_{\mathbb{R}^{3 \times 3}}\left(|G w|^{2}-\left|F_{\lambda} w\right|^{2}\right) d \nu_{x}(G) d x \\
& =\frac{1}{\operatorname{meas} \Omega} \int_{\Omega} \int_{\mathbb{R}^{3 \times 3}}\left|\left(G-F_{\lambda}\right) w\right|^{2} d \nu_{x}(G) d x \geq 0 .
\end{aligned}
$$

But the rank-one connection (2.7) and the definition of $F_{\lambda}$ (2.10) imply that

$$
\left|F_{\lambda} w\right|=\left|U_{i} w\right|=\left|U_{j} w\right| \quad \forall w \in \mathbb{R}^{3}, w \cdot n=0 .
$$

This, together with (3.14), implies that

$$
\rho_{1}(w)=\sum_{k \neq i, j} \tau_{k}\left(\left|U_{k} w\right|^{2}-\left|F_{\lambda} w\right|^{2}\right) \geq 0 \quad \forall w \in \mathbb{R}^{3}, w \cdot n=0 .
$$

By a similar argument, we have that

$$
\begin{aligned}
\rho_{2}(w) & :=\sum_{k=1}^{6} \tau_{k}\left[\left|\left(\operatorname{Cof} U_{k}\right) w\right|^{2}-\left|\left(\operatorname{Cof} F_{\lambda}\right) w\right|^{2}\right] \\
& =\frac{1}{\operatorname{meas} \Omega} \int_{\Omega} \int_{\mathbb{R}^{3 \times 3}}\left|\left[\operatorname{Cof} G-\operatorname{Cof} F_{\lambda}\right] w\right|^{2} d \nu_{x}(G) d x \geq 0 \quad \forall w \in \mathbb{R}^{3} .
\end{aligned}
$$

Recall that the cofactor of a nonsingular matrix $A \in \mathbb{R}^{3 \times 3}$ is given by Cof $A=$ $\operatorname{det}(A) A^{-T}$. A simple calculation based on (2.7), together with the fact that $\operatorname{det}\left(Q U_{i}\right)=$ $\operatorname{det} U_{j}>0$, then leads to the rank-one connection of cofactors

$$
\operatorname{Cof}\left(Q U_{i}\right)-\operatorname{Cof} U_{j}=-\left(\operatorname{det} U_{i}\right)\left(Q U_{i}\right)^{-T} n \otimes U_{j}^{-1} a
$$


Moreover, since the cofactor is rank-one affine (cf. $[4,5,18])$, we have by $(2.7)$ and (2.10) that

$$
\operatorname{Cof} F_{\lambda}=\lambda \operatorname{Cof}\left(Q U_{i}\right)+(1-\lambda) \operatorname{Cof} U_{j}
$$

Therefore, we can conclude that

$$
\left|\left(\operatorname{Cof} F_{\lambda}\right) w\right|=\left|\left(\operatorname{Cof} U_{i}\right) w\right|=\left|\left(\operatorname{Cof} U_{j}\right) w\right| \quad \forall w \in \mathbb{R}^{3}, w \cdot U_{j}^{-1} a=0,
$$

which, together with (3.17), implies that

$$
\rho_{2}(w)=\sum_{k \neq i, j} \tau_{k}\left[\left|\left(\operatorname{Cof} U_{k}\right) w\right|^{2}-\left|\left(\operatorname{Cof} F_{\lambda}\right) w\right|^{2}\right] \geq 0 \quad \forall w \in \mathbb{R}^{3}, w \cdot U_{j}^{-1} a=0
$$

We will use the two inequalities (3.16) and (3.19) with special choices of $w \in \mathbb{R}^{3}$ to prove our result of uniqueness.

(1) Compound twinning. Without loss of generality, we may assume that $(i, j)=$ $(1,2)$. In this case, there are two distinct (up to scalar multiples) normals $n=e_{2}$ and $n=e_{3}$ that solve the twinning equation (2.7), cf. Lemma 2.2. We only consider the case $n=e_{2}$, since the other case can be treated similarly. In this case, $U_{2}^{-1} a$ is parallel to $e_{3}$. We first prove the uniqueness of the Young measure for parameters that do not satisfy (3.4).

Setting $w=e_{1}$ and $w=e_{3}$ in (3.16), we obtain by a series of calculations using (2.4) and (2.10) that

$$
\rho_{1}\left(e_{1}\right)=\left(\tau_{3}+\tau_{4}+\tau_{5}+\tau_{6}\right)\left(\xi^{2}+\eta^{2}-\zeta^{2}\right) \geq 0
$$

and

$$
\rho_{1}\left(e_{3}\right)=\left(\tau_{5}+\tau_{6}\right)\left(\zeta^{2}-\xi^{2}-\eta^{2}\right) \geq 0,
$$

respectively. Consequently, we have

$$
\tau_{3}=\tau_{4}=\tau_{5}=\tau_{6}=0 \quad \text { if } \quad \zeta^{2}>\xi^{2}+\eta^{2}
$$

and

$$
\tau_{5}=\tau_{6}=0 \quad \text { if } \quad \zeta^{2}<\xi^{2}+\eta^{2} .
$$

Similarly, setting $w=e_{1}$ and $w=e_{2}$ in (3.19), we can obtain

$$
\rho_{2}\left(e_{1}\right)=\left(\tau_{3}+\tau_{4}+\tau_{5}+\tau_{6}\right)\left[\zeta^{2}\left(\xi^{2}+\eta^{2}\right)-\left(\xi^{2}-\eta^{2}\right)^{2}\right] \geq 0
$$

and

$$
\rho_{2}\left(e_{2}\right)=\left(\tau_{3}+\tau_{4}\right)\left[\left(\xi^{2}-\eta^{2}\right)^{2}-\zeta^{2}\left(\xi^{2}+\eta^{2}\right)\right] \geq 0
$$

leading to

$$
\tau_{3}=\tau_{4}=\tau_{5}=\tau_{6}=0 \quad \text { if } \quad \zeta^{2}<\frac{\left(\xi^{2}-\eta^{2}\right)^{2}}{\xi^{2}+\eta^{2}}
$$

and

$$
\tau_{3}=\tau_{4}=0 \quad \text { if } \quad \zeta^{2}>\frac{\left(\xi^{2}-\eta^{2}\right)^{2}}{\xi^{2}+\eta^{2}}
$$


respectively. It follows now from $(3.22),(3.23),(3.26)$, and $(3.27)$ that $\tau_{3}=\tau_{4}=\tau_{5}=$ $\tau_{6}=0$ for all $\xi, \eta$, and $\zeta$ except those that satisfy $(3.4)$.

We now show that if $\xi, \eta$, and $\zeta$ satisfy (3.4), then the Young measure is not unique for $\lambda=1 / 2$. To this end, we first calculate $F_{\lambda}^{T} F_{\lambda}$. For our choice that $(i, j)=(1,2)$ and $n=e_{2}$, we can obtain from (2.8) that

$$
a=\frac{4 \xi \eta}{\xi^{2}+\eta^{2}}(0,-\eta, \xi)
$$

A series of calculations then yield that

$$
F_{\lambda}^{T} F_{\lambda}=\left(\begin{array}{ccc}
\zeta^{2} & 0 & 0 \\
0 & \sigma(\lambda) & 2(2 \lambda-1) \xi \eta \\
0 & 2(2 \lambda-1) \xi \eta & \xi^{2}+\eta^{2}
\end{array}\right)
$$

where

$$
\sigma(\lambda)=\xi^{2}+\eta^{2}+\frac{16 \xi^{2} \eta^{2}}{\xi^{2}+\eta^{2}} \lambda(\lambda-1) .
$$

Setting $\lambda=1 / 2$, we then obtain that

$$
F_{1 / 2}^{T} F_{1 / 2}=\left(\begin{array}{ccc}
\zeta^{2} & 0 & 0 \\
0 & \frac{\left(\xi^{2}-\eta^{2}\right)^{2}}{\xi^{2}+\eta^{2}} & 0 \\
0 & 0 & \xi^{2}+\eta^{2}
\end{array}\right)
$$

Now set

$$
G_{\lambda}=\lambda \bar{Q} U_{3}+(1-\lambda) U_{4} \quad \text { and } \quad H_{\lambda}=\lambda \tilde{Q} U_{5}+(1-\lambda) U_{6},
$$

where $\bar{Q} \in \mathrm{SO}(3)$ and $\tilde{Q} \in \mathrm{SO}(3)$ are chosen to satisfy the twinning equation (2.7) for the variant pairs given by $(i, j)=(3,4)$ with the normal $n=e_{1}$ and $(i, j)=(5,6)$ with the normal $n=e_{2}$, respectively. Either by direct calculations or by (3.29) using the symmetry described in Lemma 2.1 , we can obtain for $\lambda=1 / 2$ that

$$
G_{1 / 2}^{T} G_{1 / 2}=\left(\begin{array}{ccc}
\frac{\left(\xi^{2}-\eta^{2}\right)^{2}}{\xi^{2}+\eta^{2}} & 0 & 0 \\
0 & \zeta^{2} & 0 \\
0 & 0 & \xi^{2}+\eta^{2}
\end{array}\right), H_{1 / 2}^{T} H_{1 / 2}=\left(\begin{array}{ccc}
\xi^{2}+\eta^{2} & 0 & 0 \\
0 & \frac{\left(\xi^{2}-\eta^{2}\right)^{2}}{\xi^{2}+\eta^{2}} & 0 \\
0 & 0 & \zeta^{2}
\end{array}\right)
$$

If $\zeta^{2}=\left(\xi^{2}-\eta^{2}\right)^{2} /\left(\xi^{2}+\eta^{2}\right)$, then $F_{1 / 2}^{T} F_{1 / 2}=G_{1 / 2}^{T} G_{1 / 2}$. This implies that there exists $R \in \mathrm{SO}(3)$ such that $F_{1 / 2}=R G_{1 / 2}$. We can thus conclude that, in addition to the one given in (3.3) with $i=1, j=2$, and $\lambda=1 / 2$, there is another Young measure given by

$$
\nu_{x}=\frac{1}{2} \delta_{R \bar{Q} U_{3}}+\frac{1}{2} \delta_{R U_{4}} \quad \text { a.e. } x \in \Omega .
$$

Thus, the Young measure is not unique. If on the other hand $\zeta^{2}=\xi^{2}+\eta^{2}$, we see that $F_{1 / 2}^{T} F_{1 / 2}=H_{1 / 2}^{T} H_{1 / 2}$, and once again conclude that the Young measure is not unique.

(2) Noncompound twinning. Without loss of generality, we may assume that $(i, j)=$ $(1,3)$. According to Lemma 2.1 and Lemma 2.2, the solutions to the twinning equation 
(2.7) are given by $(2.8)$ and $(2.9)$ with $e=(1 / \sqrt{2})(1,-1,0)$. An easy calculation shows that, up to multiplication by a scalar,

$$
n_{1}=\frac{1}{\sqrt{2}}(1,-1,0) \quad \text { and } \quad U_{3}^{-1} a_{2}=\frac{1}{\sqrt{2}}(1,-1,0) .
$$

We first consider a type I twin. Notice from (3.31) that any vector $w \in \mathbb{R}^{3}$ such that $w \cdot n_{1}=0$ has the form that $w=(s, s, t)$ with $s, t \in \mathbb{R}$. Denoting $g_{1}(s, t):=\rho_{1}(w)$ with $w=(s, s, t)$, we get by a series of calculations using (2.4) and (2.10) in (3.16) that

$$
\begin{aligned}
& g_{1}(s, t)=(\left.\tau_{2}+\tau_{4}\right)(-8 \xi \eta s t) \\
&+\tau_{5}\left[\left(\xi^{2}+\eta^{2}-\zeta^{2}+4 \xi \eta\right) s^{2}+\left(\zeta^{2}-\xi^{2}-\eta^{2}\right) t^{2}-4 \xi \eta s t\right] \\
&+\tau_{6}\left[\left(\xi^{2}+\eta^{2}-\zeta^{2}-4 \xi \eta\right) s^{2}+\left(\zeta^{2}-\xi^{2}-\eta^{2}\right) t^{2}-4 \xi \eta s t\right] \\
& \geq 0 \quad \forall s, t \in \mathbb{R} .
\end{aligned}
$$

Setting $s=t=1$ in the above inequality, we obtain $\left(\tau_{2}+\tau_{4}+\tau_{6}\right)(-8 \xi \eta) \geq 0$ and conclude that $\tau_{2}=\tau_{4}=\tau_{6}=0$. Therefore, (3.32) reduces to $g_{1}(s, t)=\tau_{5} l_{1}(s, t) \geq 0$ for all $s, t \in \mathbb{R}$, where

$$
l_{1}(s, t)=\left(\xi^{2}+\eta^{2}-\zeta^{2}+4 \xi \eta\right) s^{2}+\left(\zeta^{2}-\xi^{2}-\eta^{2}\right) t^{2}-4 \xi \eta s t, \quad s, t \in \mathbb{R} .
$$

However, the discriminant of the quadratic form $l_{1}$ is

$$
(-4 \xi \eta)^{2}-4\left(\xi^{2}+\eta^{2}-\zeta^{2}+4 \xi \eta\right)\left(\zeta^{2}-\xi^{2}-\eta^{2}\right)=4\left[(\xi+\eta)^{2}-\zeta^{2}\right]^{2} .
$$

This is always positive except for those $\xi, \eta$, and $\zeta$ satisfying (3.5). Therefore, if $\zeta \neq \xi+\eta$, one can always choose nonzero $s_{1}, t_{1} \in \mathbb{R}$ such that $l_{1}\left(s_{1}, t_{1}\right)<0$. Since $g_{1}: \mathbb{R}^{2} \rightarrow \mathbb{R}$ is always nonnegative by (3.32), we conclude that $\tau_{5}=0$. Consequently, the Young measure is unique for a type I twin if the parameters do not satisfy (3.5).

Now suppose the parameters satisfy (3.5), that is, $\zeta=\xi+\eta$. Set $K_{\lambda}=\lambda \hat{Q} U_{5}+$ $(1-\lambda) U_{3}$ with $\hat{Q}=\hat{Q}(\lambda) \in \mathrm{SO}(3)$ so chosen to give the type I twin solution to (2.7) for the variant pair given by $(i, j)=(5,3)$. By a series of calculations (which can easily be carried out with the help of a program like Mathematica), we obtain that $F_{\lambda}^{T} F_{\lambda}=K_{\lambda}^{T} K_{\lambda}$ if $\lambda=\frac{1}{3}\left(4-\frac{\eta}{\xi}-\frac{\xi}{\eta}\right) \in(0,1)$. Following an argument similar to that used in the case of compound twins, we can thus conclude that the Young measure is not unique in this case.

We now turn to a type II twin. Notice from (3.31) that any vector $w \in \mathbb{R}^{3}$ such that $w \cdot U_{3}^{-1} a_{2}=0$ has the form that $w=(s, s, t)$ with $s, t \in \mathbb{R}$. Denoting $g_{2}(s, t):=\rho_{2}(w)$ with $w=(s, s, t)$, we get by a series of calculations using (2.4) and (2.10) in (3.19) that

$$
\begin{aligned}
& g_{2}(s, t)=(\left.\tau_{2}+\tau_{4}\right) 8 \xi \eta \zeta^{2} s t \\
&+\tau_{5}(s-t)\left\{\left[\left(\xi^{2}+\eta^{2}\right) \zeta^{2}-\left(\xi^{2}-\eta^{2}\right)^{2}\right](s+t)-4 \xi \eta \zeta^{2} s\right\} \\
&+\tau_{6}(s+t)\left\{\left[\left(\xi^{2}+\eta^{2}\right) \zeta^{2}-\left(\xi^{2}-\eta^{2}\right)^{2}\right](s-t)+4 \xi \eta \zeta^{2} s\right\} \\
& \geq 0 \quad \forall s, t \in \mathbb{R} .
\end{aligned}
$$


Setting $s=-t=1$ above, we obtain $\left(\tau_{2}+\tau_{4}+\tau_{5}\right)\left(-8 \xi \eta \zeta^{2}\right) \geq 0$ and conclude that $\tau_{2}=\tau_{4}=\tau_{5}=0$. Therefore (3.34) reduces to $g_{2}(s, t)=\tau_{6} l_{2}(s, t) \geq 0$ for all $s, t \in \mathbb{R}$, where

$$
\begin{aligned}
l_{2}(s, t)= & (s+t)\left\{\left[\left(\xi^{2}+\eta^{2}\right) \zeta^{2}-\left(\xi^{2}-\eta^{2}\right)^{2}\right](s-t)+4 \xi \eta \zeta^{2} s\right\} \\
= & {\left[\left(\xi^{2}+\eta^{2}\right) \zeta^{2}-\left(\xi^{2}-\eta^{2}\right)^{2}+4 \xi \eta \zeta^{2}\right] s^{2} } \\
& +\left[\left(\xi^{2}-\eta^{2}\right)^{2}-\left(\xi^{2}+\eta^{2}\right) \zeta^{2}\right] t^{2}+4 \xi \eta \zeta^{2} s t, \quad s, t \in \mathbb{R} .
\end{aligned}
$$

Since the discriminant of the quadratic form $l_{2}$ is

$$
\begin{aligned}
& \left(4 \xi \eta \zeta^{2}\right)^{2}-4\left[\left(\xi^{2}+\eta^{2}\right) \zeta^{2}-\left(\xi^{2}-\eta^{2}\right)^{2}+4 \xi \eta \zeta^{2}\right]\left[\left(\xi^{2}-\eta^{2}\right)^{2}-\left(\xi^{2}+\eta^{2}\right) \zeta^{2}\right] \\
& =4(\xi+\eta)^{4}\left[(\xi-\eta)^{2}-\zeta^{2}\right]^{2}
\end{aligned}
$$

we can argue as in the case of a type I twin that $\tau_{6}=0$, implying that the Young measure is unique for a type II twin, if the parameters do not satisfy (3.6). Finally, if the parameters satisfy (3.6), we can show as before that the Young measure is not unique by considering a suitable solution to the twinning equation (2.7) for the variant pair given by $(i, j)=(6,3)$. This completes the proof.

\section{A Stability Theory for a Simply Laminated Microstructure}

In addition to the assumptions (2.2) and (2.3), we assume in this section that the continuous energy density $\phi: \mathbb{R}^{3 \times 3} \rightarrow \mathbb{R}$ also satisfies the following condition on the local ellipticity and the global growth rate

$$
\phi(F) \geq \kappa\|F-\pi(F)\|^{2} \quad \forall F \in \mathbb{R}^{3 \times 3},
$$

where $\kappa>0$ is a constant and $\pi: \mathbb{R}^{3 \times 3} \rightarrow \mathcal{U}$ is the projection defined by

$$
\|F-\pi(F)\|=\min _{G \in \mathcal{U}}\|F-G\| \quad \forall F \in \mathbb{R}^{3 \times 3} .
$$

Since $\mathcal{U} \subset \mathbb{R}^{3 \times 3}$ is compact, the projection $\pi(F)$ exists for any $F \in \mathbb{R}^{3 \times 3}$, although it may not be unique. If $\pi(F) \in \mathcal{U}=\mathcal{U}_{1} \cup \cdots \cup \mathcal{U}_{6}$ for some $F \in \mathbb{R}^{3 \times 3}$ is multiply valued, we redefine its unique value to be in $\mathcal{U}_{i}$ with the smallest possible index $i$.

We define in what follows the set of admissible deformations to be

$$
\mathcal{A}:=W_{\lambda}^{1,2}\left(\Omega ; \mathbb{R}^{3}\right)=\left\{y \in W^{1,2}\left(\Omega ; \mathbb{R}^{3}\right): y(x)=F_{\lambda} x \text { for } x \in \partial \Omega\right\} .
$$

The following lemma is a direct consequence of the condition (4.1).

Lemma 4.1. We have that

$$
\int_{\Omega}\|\nabla y(x)-\pi(\nabla y(x))\|^{2} d x \leq \kappa^{-1} \mathcal{E}(y) \quad \forall y \in \mathcal{A} .
$$


In the rest of this paper, we shall be concerned only with parameters $\xi, \eta$, and $\zeta$ for which we have the uniqueness of the Young measure as in Theorem 3.1. We thus make the standing assumption that the parameters $\xi, \eta$, and $\zeta$ satisfy (2.5) and also satisfy

$$
\zeta^{2} \neq \xi^{2}+\eta^{2} \quad \text { and } \quad \zeta^{2} \neq \frac{\left(\xi^{2}-\eta^{2}\right)^{2}}{\xi^{2}+\eta^{2}}
$$

when considering a compound twin, or

$$
\zeta \neq \xi+\eta
$$

when considering a type I noncompound twin, or

$$
\zeta \neq \xi-\eta
$$

when considering a type II noncompound twin.

We shall also denote by $C$ a generic positive constant which may vary with the context. Unless otherwise indicated, this constant $C$ is assumed to be independent of $y \in \mathcal{A}$ but may be dependent on $\xi, \eta, \zeta, \lambda, \kappa$, and $\Omega$.

4.1. Estimates for the reduction of the six-well problem to a two-well problem. For each $k \in\{1, \cdots, 6\}$ and each $y \in \mathcal{A}$, we denote

$$
\Omega_{k}(y):=\left\{x \in \Omega: \pi(\nabla y(x)) \in \mathcal{U}_{k}\right\}
$$

and

$$
\tau_{k}(y):=\frac{\operatorname{meas} \Omega_{k}(y)}{\operatorname{meas} \Omega}
$$

cf. (3.12). Notice that $\tau_{k}(y)$ is the volume fraction of the deformation gradient $\nabla y$ with respect to the $k$-th energy well $\mathcal{U}_{k}$, and that (cf. (3.13))

$$
\sum_{k=1}^{6} \tau_{k}(y)=1 \quad \forall y \in \mathcal{A} \text {. }
$$

Theorem 4.1. We have

$$
\tau_{k}(y) \leq C\left[\mathcal{E}(y)^{1 / 2}+\mathcal{E}(y)\right] \quad \forall k \in\{1, \cdots, 6\} \backslash\{i, j\}, \quad \forall y \in \mathcal{A} .
$$

Proof. Fix $y \in \mathcal{A}$. We shall translate the argument using the Young measure in the proof of Theorem 3.1 into that using the projection $\pi: \mathbb{R}^{3 \times 3} \rightarrow \mathcal{U}$. We have by (4.6) and (3.8) that for any $w \in \mathbb{R}^{3}$ with $|w|=1$ (cf. (3.14))

$$
\begin{aligned}
\rho_{1}(y ; w):= & \sum_{k=1}^{6} \tau_{k}(y)\left(\left|U_{k} w\right|^{2}-\left|F_{\lambda} w\right|^{2}\right) \\
= & \frac{1}{\operatorname{meas} \Omega} \int_{\Omega}\left[|\pi(\nabla y(x)) w|^{2}-\left|F_{\lambda} w\right|^{2}\right] d x \\
= & \frac{1}{\operatorname{meas} \Omega} \int_{\Omega}\left|\left[\pi(\nabla y(x))-F_{\lambda}\right] w\right|^{2} d x \\
& \quad-\frac{2}{\operatorname{meas} \Omega} \int_{\Omega}[\nabla y(x)-\pi(\nabla y(x))] w \cdot F_{\lambda} w d x
\end{aligned}
$$




$$
\geq-\frac{2}{\operatorname{meas} \Omega} \int_{\Omega}[\nabla y(x)-\pi(\nabla y(x))] w \cdot F_{\lambda} w d x .
$$

It follows from the Cauchy-Schwarz inequality and Lemma 4.1 that

$$
\left|\frac{2}{\operatorname{meas} \Omega} \int_{\Omega}[\nabla y(x)-\pi(\nabla y(x))] w \cdot F_{\lambda} w d x\right| \leq C \mathcal{E}(y)^{1 / 2} .
$$

Thus, we obtain from (4.8) that for all $w \in \mathbb{R}^{3}$ with $|w|=1$

$$
\rho_{1}(y ; w):=\sum_{k=1}^{6} \tau_{k}(y)\left(\left|U_{k} w\right|^{2}-\left|F_{\lambda} w\right|^{2}\right) \geq-C \mathcal{E}(y)^{1 / 2},
$$

which, together with (3.15) leads to (cf. (3.16))

$$
\begin{aligned}
\rho_{1}(y ; w) & :=\sum_{k \neq i, j} \tau_{k}(y)\left(\left|U_{k} w\right|^{2}-\left|F_{\lambda} w\right|^{2}\right) \\
& \geq-C \mathcal{E}(y)^{1 / 2} \quad \forall w \in \mathbb{R}^{3},|w|=1, w \cdot n=0 .
\end{aligned}
$$

Similarly, we have by (4.6) and (3.8) that for any $w \in \mathbb{R}^{3}$ with $|w|=1$ that (cf. $(3.17))$

$$
\begin{aligned}
\rho_{2}(y ; w):= & \sum_{k=1}^{6} \tau_{k}(y)\left[\left|\left(\operatorname{Cof} U_{k}\right) w\right|^{2}-\left|\left(\operatorname{Cof} F_{\lambda}\right) w\right|^{2}\right] \\
= & \frac{1}{\operatorname{meas} \Omega} \int_{\Omega}\left[\mid\left(\left.\operatorname{Cof} \pi(\nabla y(x)) w\right|^{2}-\left|\left(\operatorname{Cof} F_{\lambda}\right) w\right|^{2}\right] d x\right. \\
= & \frac{1}{\operatorname{meas} \Omega} \int_{\Omega}\left|\left[\operatorname{Cof} \pi(\nabla y(x))-\operatorname{Cof} F_{\lambda}\right] w\right|^{2} d x \\
& -\frac{2}{\operatorname{meas} \Omega} \int_{\Omega}[\operatorname{Cof} \nabla y(x)-\operatorname{Cof} \pi(\nabla y(x))] w \cdot\left(\operatorname{Cof} F_{\lambda}\right) w d x \\
\geq & -\frac{2}{\operatorname{meas} \Omega} \int_{\Omega}[\operatorname{Cof} \nabla y(x)-\operatorname{Cof} \pi(\nabla y(x))] w \cdot\left(\operatorname{Cof} F_{\lambda}\right) w d x
\end{aligned}
$$

If we denote $V(x):=\pi(\nabla y(x))$ for any $x \in \Omega$, then $V=\left(V_{k l}\right) \in L^{\infty}\left(\Omega ; \mathbb{R}^{3 \times 3}\right)$ and its $L^{\infty}$ norm is always bounded above by a constant $C$. If we also denote $W(x):=\nabla y(x)$ for $x \in \Omega$, then $W=\left(W_{k l}\right) \in L^{2}\left(\Omega ; \mathbb{R}^{3 \times 3}\right)$. Moreover, one can easily verify for any $k, l, p, q \in\{1,2,3\}$ with $k \neq p$ and $l \neq q$ that

$$
\begin{aligned}
& \left(V_{k l} V_{p q}-V_{k q} V_{p l}\right)-\left(W_{k l} W_{p q}-W_{k q} W_{p l}\right) \\
& =V_{k l}\left(V_{p q}-W_{p q}\right)-V_{k q}\left(V_{p l}-W_{p l}\right)-V_{p l}\left(V_{k q}-W_{k q}\right)+V_{p q}\left(V_{k l}-W_{k l}\right) \\
& \quad-\left(V_{k l}-W_{k l}\right)\left(V_{p q}-W_{p q}\right)+\left(V_{k q}-W_{k q}\right)\left(V_{p l}-W_{p l}\right) .
\end{aligned}
$$

Hence, we have by the Cauchy-Schwarz inequality that

$$
\begin{gathered}
\int_{\Omega}\left|\left[V_{k l}(x) V_{p q}(x)-V_{k q}(x) V_{p l}(x)\right]-\left[W_{k l}(x) W_{p q}(x)-W_{k q}(x) W_{p l}(x)\right]\right| d x \\
\leq C\left(\|V\|_{L^{\infty}\left(\Omega ; \mathbb{R}^{3 \times 3}\right)}\|V-W\|_{L^{2}\left(\Omega ; \mathbb{R}^{3 \times 3}\right)}+\|V-W\|_{L^{2}\left(\Omega ; \mathbb{R}^{3 \times 3}\right)}^{2}\right) .
\end{gathered}
$$


The definition of the cofactor matrix and Lemma 4.1 then imply that

$$
\int_{\Omega}|[\operatorname{Cof} \nabla y(x)-\operatorname{Cof} \pi(\nabla y(x))] w| d x \leq C\left[\mathcal{E}(y)^{1 / 2}+\mathcal{E}(y)\right] .
$$

It thus follows from (4.11) and (4.12) that

$$
\begin{aligned}
\rho_{2}(y ; w) & :=\sum_{k=1}^{6} \tau_{k}(y)\left[\left|\left(\operatorname{Cof} U_{k}\right) w\right|^{2}-\left|\left(\operatorname{Cof} F_{\lambda}\right) w\right|^{2}\right] \\
& \geq-C\left[\mathcal{E}(y)^{1 / 2}+\mathcal{E}(y)\right] \quad \forall w \in \mathbb{R}^{3},|w|=1
\end{aligned}
$$

which, together with (3.18), leads to (cf. (3.19))

$$
\begin{aligned}
\rho_{2}(y ; w) & :=\sum_{k \neq i, j} \tau_{k}(y)\left[\left|\left(\operatorname{Cof} U_{k}\right) w\right|^{2}-\left|\left(\operatorname{Cof} F_{\lambda}\right) w\right|^{2}\right] \\
& \geq-C\left[\mathcal{E}(y)^{1 / 2}+\mathcal{E}(y)\right] \quad \forall w \in \mathbb{R}^{3},|w|=1, w \cdot U_{j}^{-1} a=0 .
\end{aligned}
$$

Case 1. Compound twinning. Without loss of generality, we may assume as in the proof of Theorem 3.1 that $(i, j)=(1,2)$ and $n=e_{2}$.

Setting $w=e_{1}$ in (4.10), we obtain (cf. (3.20))

$$
\rho_{1}\left(y ; e_{1}\right)=\left[\tau_{3}(y)+\tau_{4}(y)+\tau_{5}(y)+\tau_{6}(y)\right]\left(\xi^{2}+\eta^{2}-\zeta^{2}\right) \geq-C \mathcal{E}(y)^{1 / 2}
$$

leading to (cf. (3.22))

$$
\tau_{3}(y)+\tau_{4}(y)+\tau_{5}(y)+\tau_{6}(y) \leq C \mathcal{E}(y)^{1 / 2} \quad \text { if } \quad \zeta^{2}>\xi^{2}+\eta^{2} .
$$

Setting $w=e_{3}$ in (4.10), we obtain that (cf. (3.21))

$$
\rho_{1}\left(y ; e_{3}\right)=\left[\tau_{5}(y)+\tau_{6}(y)\right]\left(\zeta^{2}-\xi^{2}-\eta^{2}\right) \geq-C \mathcal{E}(y)^{1 / 2},
$$

so (cf. $(3.23))$

$$
\tau_{5}(y)+\tau_{6}(y) \leq C \mathcal{E}(y)^{1 / 2} \quad \text { if } \quad \zeta^{2}<\xi^{2}+\eta^{2} .
$$

Similarly, setting $w=e_{1}$ and $w=e_{2}$ in (4.13), we obtain that (cf. (3.24) $-(3.27)$ )

$$
\begin{aligned}
\rho_{2}\left(y ; e_{1}\right) & =\left[\tau_{3}(y)+\tau_{4}(y)+\tau_{5}(y)+\tau_{6}(y)\right]\left[\zeta^{2}\left(\xi^{2}+\eta^{2}\right)-\left(\xi^{2}-\eta^{2}\right)^{2}\right] \\
& \geq-C\left[\mathcal{E}(y)^{1 / 2}+\mathcal{E}(y)\right]
\end{aligned}
$$

and

$$
\begin{aligned}
\rho_{2}\left(y ; e_{2}\right) & =\left[\tau_{3}(y)+\tau_{4}(y)\right]\left[\left(\xi^{2}-\eta^{2}\right)^{2}-\zeta^{2}\left(\xi^{2}+\eta^{2}\right)\right] \\
& \geq-C\left[\mathcal{E}(y)^{1 / 2}+\mathcal{E}(y)\right]
\end{aligned}
$$

leading to

$$
\tau_{3}(y)+\tau_{4}(y)+\tau_{5}(y)+\tau_{6}(y) \leq C\left[\mathcal{E}(y)^{1 / 2}+\mathcal{E}(y)\right] \quad \text { if } \quad \zeta^{2}<\frac{\left(\xi^{2}-\eta^{2}\right)^{2}}{\xi^{2}+\eta^{2}}
$$


and

$$
\tau_{3}(y)+\tau_{4}(y) \leq C\left[\mathcal{E}(y)^{1 / 2}+\mathcal{E}(y)\right] \quad \text { if } \quad \zeta^{2}>\frac{\left(\xi^{2}-\eta^{2}\right)^{2}}{\xi^{2}+\eta^{2}}
$$

respectively. We thus can conclude from $(4.14)-(4.17)$ that

$$
\tau_{3}(y)+\tau_{4}(y)+\tau_{5}(y)+\tau_{6}(y) \leq C\left[\mathcal{E}(y)^{1 / 2}+\mathcal{E}(y)\right]
$$

for all $\xi, \eta$, and $\zeta$ that satisfy (4.3).

Case 2. Noncompound twinning. Without loss of generality, we may assume as in the proof of Theorem 3.1 that $(i, j)=(1,3)$. The solutions to the twinning equation (2.7) are given by $(2.8)$ and $(2.9)$ with $e=(1 / \sqrt{2})(1,-1,0)$. We also have (cf. (3.31))

$$
n_{1}=\frac{1}{\sqrt{2}}(1,-1,0) \text { and } \quad U_{3}^{-1} a_{2}=\frac{1}{\sqrt{2}}(1,-1,0) .
$$

We first consider a type I noncompound twin, that is, the rank-one connection with $n=n_{1}=e=\frac{1}{\sqrt{2}}(1,-1,0)$. Notice that any vector $w \in \mathbb{R}^{3}$ such that $w \cdot n_{1}=0$ has the form $w=(s, s, t)$ with $s, t \in \mathbb{R}$. If we denote $g_{1}(y ; s, t):=\rho_{1}(y ; w)$ with $w=(s, s, t)$, then we have by (4.10) and by the same calculation as performed in (3.32) that

$$
\begin{aligned}
g_{1}(y ; s, t)=[ & \left.\tau_{2}(y)+\tau_{4}(y)\right](-8 \xi \eta s t) \\
& +\tau_{5}(y)\left[\left(\xi^{2}+\eta^{2}-\zeta^{2}+4 \xi \eta\right) s^{2}+\left(\zeta^{2}-\xi^{2}-\eta^{2}\right) t^{2}-4 \xi \eta s t\right] \\
& +\tau_{6}(y)\left[\left(\xi^{2}+\eta^{2}-\zeta^{2}-4 \xi \eta\right) s^{2}+\left(\zeta^{2}-\xi^{2}-\eta^{2}\right) t^{2}-4 \xi \eta s t\right] \\
\geq & -C \mathcal{E}(y)^{1 / 2} \quad \forall s, t \in \mathbb{R} .
\end{aligned}
$$

Setting $s=t=1$ in (4.18), we obtain

$$
\tau_{2}(y)+\tau_{4}(y)+\tau_{6}(y) \leq C \mathcal{E}(y)^{1 / 2} .
$$

Notice that the coefficient of $\tau_{5}(y)$ in (4.18) is precisely $l_{1}(s, t)$ which is defined in (3.33). Since $\zeta \neq \xi+\eta$ in this case, one can again always choose nonzero $s_{1}, t_{1} \in \mathbb{R}$ such that $l_{1}\left(s_{1}, t_{1}\right)<0$ as argued before. Setting $s=s_{1}$ and $t=t_{1}$ in (4.18), we obtain using the estimate (4.19) that

$$
\tau_{5}(y) \leq C \mathcal{E}(y)^{1 / 2}
$$

We now consider a type II noncompound twin. By (4.13) and a series of calculations similar to (3.34), we can obtain for $g_{2}(y ; s, t):=\rho_{2}(w)$ with $w=(s, s, t)$ and $s, t \in \mathbb{R}$ that

$$
\begin{aligned}
g_{2}(y ; s, t)=[ & \left.\tau_{2}(y)+\tau_{4}(y)\right] 8 \xi \eta \zeta^{2} s t \\
& +\tau_{5}(y)(s-t)\left\{\left[\left(\xi^{2}+\eta^{2}\right) \zeta^{2}-\left(\xi^{2}-\eta^{2}\right)^{2}\right](s+t)-4 \xi \eta \zeta^{2} s\right\} \\
& +\tau_{6}(y)(s+t)\left\{\left[\left(\xi^{2}+\eta^{2}\right) \zeta^{2}-\left(\xi^{2}-\eta^{2}\right)^{2}\right](s-t)+4 \xi \eta \zeta^{2} s\right\} \\
\geq- & C\left[\mathcal{E}(y)^{1 / 2}+\mathcal{E}(y)\right] \quad \forall s, t \in \mathbb{R} .
\end{aligned}
$$


Setting $s=-t=1$ in $(4.20)$, we get

$$
\tau_{2}(y)+\tau_{4}(y)+\tau_{5}(y) \leq C\left[\mathcal{E}(y)^{1 / 2}+\mathcal{E}(y)\right] .
$$

Notice that the coefficient of the term $\tau_{6}(y)$ in (4.20) is precisely the quadratic form $l_{2}(s, t)$ defined in (3.35). Thus, by the same argument as in proving $\tau_{6}=0$ at the end of the proof of Theorem 4.1, we can choose nonzero $s_{2}, t_{2} \in \mathbb{R}$ such that $l_{2}\left(s_{2}, t_{2}\right)<0$. With these values of $s_{2}$ and $t_{2}$, we obtain from (4.20) and (4.21) that

$$
\tau_{6}(y) \leq C\left[\mathcal{E}(y)^{1 / 2}+\mathcal{E}(y)\right]
$$

The proof is complete.

4.2. Estimates of deformations and deformation gradients. We first give a useful lemma on an estimate for directional derivatives of deformations in the direction tangential to the twin planes.

Lemma 4.2. For any $w \in \mathbb{R}^{3}$ such that $w \cdot n=0$ and $|w|=1$, we have

$$
\int_{\Omega}\left|\left[\pi(\nabla y(x))-F_{\lambda}\right] w\right|^{2} d x \leq C\left[\mathcal{E}(y)^{1 / 2}+\mathcal{E}(y)\right] \quad \forall y \in \mathcal{A} .
$$

Proof. Fix $w \in \mathbb{R}^{3}$ such that $w \cdot n=0$ and $|w|=1$. Fix also $y \in \mathcal{A}$. We have by (4.8) that

$$
\int_{\Omega}\left|\left[\pi(\nabla y(x))-F_{\lambda}\right] w\right|^{2} d x=J_{1}(y ; w)+(\operatorname{meas} \Omega) \sum_{k=1}^{6} \tau_{k}(y)\left(\left|U_{k} w\right|^{2}-\left|F_{\lambda} w\right|^{2}\right)
$$

where we have by (4.9) that

$$
\left|J_{1}(y ; w)\right|:=\left|\int_{\Omega} 2[\nabla y(x)-\pi(\nabla y(x))] w \cdot F_{\lambda} w d x\right| \leq C \mathcal{E}(y)^{1 / 2} .
$$

The desired inequality follows from (4.22), (4.23), and the reduction estimate (4.7).

Now we give a series of estimates for all the admissible deformations and their gradients. These estimates indicate that any energy-minimizing sequence of deformations converges strongly, and that the corresponding sequence of deformation gradients converges to $F_{\lambda}$ weakly in general but strongly in any direction tangential to a twin plane.

Theorem 4.2. (1) For any $w \in \mathbb{R}^{3}$ such that $w \cdot n=0$ and $|w|=1$, we have

$$
\int_{\Omega}\left|\left[\nabla y(x)-F_{\lambda}\right] w\right|^{2} d x \leq C\left[\mathcal{E}(y)^{1 / 2}+\mathcal{E}(y)\right] \quad \forall y \in \mathcal{A} .
$$

(2) We have

$$
\int_{\Omega}\left|y(x)-F_{\lambda} x\right|^{2} d x \leq C\left[\mathcal{E}(y)^{1 / 2}+\mathcal{E}(y)\right] \quad \forall y \in \mathcal{A} .
$$

(3) For any Lipschitz domain $\omega \subset \Omega$, there exists a constant $C=C(\omega)>0$ such that

$$
\left\|\int_{\omega}\left[\nabla y(x)-F_{\lambda}\right] d x\right\| \leq C\left[\mathcal{E}(y)^{1 / 8}+\mathcal{E}(y)^{1 / 2}\right] \quad \forall y \in \mathcal{A} .
$$


Proof. Fix $y \in \mathcal{A}$.

(1) If $w \in \mathbb{R}^{3}$ satisfies $w \cdot n=0$ and $|w|=1$, then the inequality

$$
\begin{aligned}
& \int_{\Omega}\left|\left[\nabla y(x)-F_{\lambda}\right] w\right|^{2} d x \\
& \quad \leq 2 \int_{\Omega}|[\nabla y(x)-\pi(\nabla y(x))] w|^{2} d x+2 \int_{\Omega}\left|\left[\pi(\nabla y(x))-F_{\lambda}\right] w\right|^{2} d x,
\end{aligned}
$$

together with Lemma 4.1 and Lemma 4.2, implies (4.24).

(2) Since $y(x)-F_{\lambda} x=0$ for $x \in \partial \Omega$ in the sense of trace, we can apply the Poincaré inequality $[33,44]$

$$
\int_{\Omega}\left|y(x)-F_{\lambda} x\right|^{2} d x \leq C \int_{\Omega}\left|\left[\nabla y(x)-F_{\lambda}\right] w\right|^{2} d x \quad \forall w \in \mathbb{R}^{3},|w|=1,
$$

with $w \in \mathbb{R}^{3}$ so chosen that $w \cdot n=0$ to obtain the inequality (4.25) from (4.24).

(3) If follows from the divergence theorem and the Cauchy-Schwarz inequality that

$$
\begin{gathered}
\left\|\int_{\omega}\left[\nabla y(x)-F_{\lambda}\right] d x\right\|=\left\|\int_{\partial \omega}\left[y(x)-F_{\lambda} x\right] \otimes \nu d S\right\| \\
\leq \int_{\partial \omega}\left|y(x)-F_{\lambda} x\right| d S \\
\leq\left(\operatorname{meas}_{2} \partial \omega\right)^{1 / 2}\left(\int_{\partial \omega}\left|y(x)-F_{\lambda} x\right|^{2} d S\right)^{1 / 2},
\end{gathered}
$$

where $\nu$ is the unit exterior normal to $\partial \omega$ and meas ${ }_{2} \partial \omega$ is the surface area of $\partial \omega$. We can obtain by the trace theorem $[3,44]$ that

$$
\begin{aligned}
\int_{\partial \omega} \mid y(x)- & \left.F_{\lambda} x\right|^{2} d S \\
\leq C & {\left[\int_{\omega}\left|y(x)-F_{\lambda} x\right|^{2} d x+\int_{\omega}\left|\nabla\left(\left|y(x)-F_{\lambda} x\right|^{2}\right)\right| d x\right] } \\
\leq C & {\left[\int_{\omega}\left|y(x)-F_{\lambda} x\right|^{2} d x\right.} \\
+ & \left.\left(\int_{\omega}\left|y(x)-F_{\lambda} x\right|^{2} d x\right)^{1 / 2}\left(\int_{\omega}\left\|\nabla y(x)-F_{\lambda}\right\|^{2} d x\right)^{1 / 2}\right] .
\end{aligned}
$$

Further, we have by the triangle inequality and Lemma 4.1 that

$$
\begin{aligned}
& \left(\int_{\omega}\left\|\nabla y(x)-F_{\lambda}\right\|^{2} d x\right)^{1 / 2} \\
& \quad \leq\left(\int_{\omega}\|\nabla y(x)-\pi(\nabla y(x))\|^{2} d x\right)^{1 / 2}+\left(\int_{\omega}\left\|\pi(\nabla y(x))-F_{\lambda}\right\|^{2} d x\right)^{1 / 2} \\
& \quad \leq C\left[\mathcal{E}(y)^{1 / 2}+1\right] .
\end{aligned}
$$


It now follows from $(4.28),(4.29),(4.25)$, and the fact $\mathcal{E}^{1 / 2} \leq \max \left(\mathcal{E}, \mathcal{E}^{1 / 4}\right) \leq \mathcal{E}+\mathcal{E}^{1 / 4}$ that

$$
\int_{\partial \omega}\left|y(x)-F_{\lambda} x\right|^{2} d S \leq C\left[\mathcal{E}(y)^{1 / 4}+\mathcal{E}(y)\right] .
$$

We finally obtain the inequality (4.26) by substituting (4.30) into (4.27).

Corollary 4.1. There does not exist any $y \in \mathcal{A}$ such that

$$
\mathcal{E}(y)=\min _{z \in \mathcal{A}} \mathcal{E}(z)
$$

Proof. If $y \in \mathcal{A}$ was a minimizer, then we would have that $\mathcal{E}(y)=0$ by Lemma 2.4 and further that $y(x)=F_{\lambda} x$ for almost every $x \in \Omega$ by (4.25). But $F_{\lambda} \notin \mathcal{U}$ by Lemma 2.3. Hence, it follows from (2.3) that

$$
0=\mathcal{E}(y)=(\operatorname{meas} \Omega) \phi\left(F_{\lambda}\right)>0,
$$

which is a contradiction.

For the fixed $i, j \in\{1, \cdots, 6\}$ with $i \neq j$, we now define the projection $\pi_{i j}: \mathbb{R}^{3 \times 3} \rightarrow$ $\mathcal{U}_{i} \cup \mathcal{U}_{j}$ by

$$
\left\|F-\pi_{i j}(F)\right\|=\min _{G \in \mathcal{U}_{i} \cup \mathcal{U}_{j}}\|F-G\| \quad \forall F \in \mathbb{R}^{3 \times 3} .
$$

If $\pi_{i j}(F)$ is multiply valued for some $F \in \mathbb{R}^{3 \times 3}$, then we redefine its unique value to be the one in $\mathcal{U}_{i}$. We also define the operators $\Theta: \mathbb{R}^{3 \times 3} \rightarrow \mathrm{SO}(3)$ and $\Pi: \mathbb{R}^{3 \times 3} \rightarrow$ $\left\{Q U_{i}, U_{j}\right\}$ by the unique decomposition

$$
\pi_{i j}(F)=\Theta(F) \Pi(F) \quad \forall F \in \mathbb{R}^{3 \times 3} .
$$

The following theorem indicates that, for any energy-minimizing sequence of deformations, the corresponding sequence of deformation gradients converges to the two homogeneous deformation gradients $Q U_{i}$ and $U_{j}$ that determine the Dirichlet boundary condition (2.12).

Theorem 4.3. We have

$$
\int_{\Omega}\|\nabla y(x)-\Pi(\nabla y(x))\|^{2} d x \leq C\left[\mathcal{E}(y)^{1 / 2}+\mathcal{E}(y)\right] \quad \forall y \in \mathcal{A} .
$$

Proof. Since $\pi_{i j}(F)=\pi(F)$ for any $F \in \mathbb{R}^{3 \times 3}$ such that $\pi(F) \in \mathcal{U}_{i} \cup \mathcal{U}_{j}$, by Lemma 4.1, Theorem 4.1, and the decomposition

$$
\nabla y-\Pi(\nabla y)=[\nabla y-\pi(\nabla y)]+\left[\pi(\nabla y)-\pi_{i j}(\nabla y)\right]+\left[\pi_{i j}(\nabla y)-\Pi(\nabla y)\right]
$$

we need only to show that

$$
\int_{\Omega}\left\|\pi_{i j}(\nabla y(x))-\Pi(\nabla y(x))\right\|^{2} d x \leq C\left[\mathcal{E}(y)^{1 / 2}+\mathcal{E}(y)\right] \quad \forall y \in \mathcal{A}
$$

Since

$$
\pi_{i j}(F)-\Pi(F)=[\Theta(F)-I] \Pi(F) \quad \forall F \in \mathbb{R}^{3 \times 3},
$$


where $I \in \mathbb{R}^{3 \times 3}$ is the identity matrix, and $\Pi(F)$ for any $F \in \mathbb{R}^{3 \times 3}$ is always bounded by a constant, we need only to show that

$$
\int_{\Omega}\|\Theta(\nabla y(x))-I\|^{2} d x \leq C\left[\mathcal{E}(y)^{1 / 2}+\mathcal{E}(y)\right] \quad \forall y \in \mathcal{A}
$$

Choose $w_{1}, w_{2} \in \mathbb{R}^{3}$ such that

$$
w_{1} \cdot n=w_{2} \cdot n=0 \quad \text { and } \quad\left|w_{1}\right|=\left|w_{2}\right|=1 .
$$

By the rank-one connection (2.7) and the definition of $F_{\lambda}$, we have

$$
\Pi(F) w_{k}=Q U_{i} w_{k}=U_{j} w_{k}=F_{\lambda} w_{k} \quad \forall F \in \mathbb{R}^{3 \times 3}, k=1,2 .
$$

This implies that

$$
[\Theta(F)-I] U_{j} w_{k}=[\Theta(F)-I] \Pi(F) w_{k}=\left[\pi_{i j}(F)-F_{\lambda}\right] w_{k} \quad \forall F \in \mathbb{R}^{3 \times 3}, k=1,2 .
$$

Setting $F=\nabla y(x), x \in \Omega$, for a fixed $y \in \mathcal{A}$, we thus obtain by Theorem 4.1 and Lemma 4.2 that

$$
\begin{aligned}
\int_{\Omega} \mid[\Theta & (\nabla y(x))-I]\left.U_{j} w_{k}\right|^{2} d x \\
& =\int_{\Omega}\left|\left[\pi_{i j}(\nabla y(x))-F_{\lambda}\right] w_{k}\right|^{2} d x \\
& \leq 2 \int_{\Omega}\left\|\pi_{i j}(\nabla y(x))-\pi(\nabla y(x))\right\|^{2} d x+2 \int_{\Omega}\left|\left[\pi(\nabla y(x))-F_{\lambda}\right] w_{k}\right|^{2} d x \\
& \leq C\left[\mathcal{E}(y)^{1 / 2}+\mathcal{E}(y)\right], \quad k=1,2 .
\end{aligned}
$$

Setting $w_{3}:=U_{j} w_{1} \times U_{j} w_{2}$, we have

$$
R w_{3}=R U_{j} w_{1} \times R U_{j} w_{2} \quad \forall R \in \mathrm{SO}(3) .
$$

One can verify that

$$
\begin{aligned}
& {[\Theta(F)-I] w_{3}=\left[\Theta(F) U_{j} w_{1} \times \Theta(F) U_{j} w_{2}\right]-\left[U_{j} w_{1} \times U_{j} w_{2}\right]} \\
& \quad=\left\{[\Theta(F)-I] U_{j} w_{1} \times \Theta(F) U_{j} w_{2}\right\}-\left\{U_{j} w_{1} \times[I-\Theta(F)] U_{j} w_{2}\right\}
\end{aligned}
$$

for all $F \in \mathbb{R}^{3 \times 3}$. Setting $F=\nabla y(x)$ for $x \in \Omega$ in this identity, we then obtain by (4.33) that

$$
\int_{\Omega}\left|[\Theta(\nabla y(x))-I] w_{3}\right|^{2} d x \leq C\left[\mathcal{E}(y)^{1 / 2}+\mathcal{E}(y)\right]
$$

Since $\left\{w_{1}, w_{2}, w_{3}\right\}$ is a basis for $\mathbb{R}^{3},(4.33)$ and (4.34) imply (4.32), hence the assertion of the theorem. 


\subsection{Estimates of volume fractions and nonlinear integrals of deformation}

gradients. For any subset $\omega \subset \Omega, \rho>0$, and $y \in \mathcal{A}$, we define the sets

$$
\begin{aligned}
& \omega_{\rho}^{i}(y)=\left\{x \in \omega: \Pi(\nabla y(x))=Q U_{i} \text { and }\left\|\nabla y(x)-Q U_{i}\right\|<\rho\right\}, \\
& \omega_{\rho}^{j}(y)=\left\{x \in \omega: \Pi(\nabla y(x))=U_{j} \text { and }\left\|\nabla y(x)-U_{j}\right\|<\rho\right\} .
\end{aligned}
$$

The following theorem states that for any Lipschitz domain $\omega \subset \Omega$ and any energyminimizing sequence $\left\{y_{k}\right\}$ in $\mathcal{A}$, the volume fraction that $\nabla y_{k}$ is near $Q U_{i}$ converges to $\lambda$ and the volume fraction that $\nabla y_{k}$ is near $U_{j}$ converges to $1-\lambda$.

Theorem 4.4. For any Lipschitz domain $\omega \subset \Omega$ and any $\rho>0$, there exists a constant $C=C(\omega, \rho)>0$ such that

$$
\left|\frac{\operatorname{meas} \omega_{\rho}^{i}(y)}{\operatorname{meas} \omega}-\lambda\right|+\left|\frac{\operatorname{meas} \omega_{\rho}^{j}(y)}{\operatorname{meas} \omega}-(1-\lambda)\right| \leq C\left[\mathcal{E}(y)^{1 / 8}+\mathcal{E}(y)^{1 / 2}\right] \quad \forall y \in \mathcal{A} .
$$

Proof. Fix $y \in \mathcal{A}$. By the definition of $\omega_{\rho}^{i}:=\omega_{\rho}^{i}(y)$ and $\omega_{\rho}^{j}:=\omega_{\rho}^{j}(y)$, we have that

$$
\begin{aligned}
& {\left[\operatorname{meas} \omega_{\rho}^{i}-\lambda \text { meas } \omega\right] Q U_{i}+\left[\operatorname{meas} \omega_{\rho}^{j}-(1-\lambda) \text { meas } \omega\right] U_{j}} \\
& =\int_{\omega}\left[\Pi(\nabla y(x))-F_{\lambda}\right] d x-\int_{\omega-\left\{\omega_{\rho}^{i} \cup \omega_{\rho}^{j}\right\}} \Pi(\nabla y(x)) d x .
\end{aligned}
$$

By the Cauchy-Schwarz inequality, Theorem 4.3, and Theorem 4.2, we obtain that

$$
\begin{aligned}
& \left\|\int_{\omega}\left[\Pi(\nabla y(x))-F_{\lambda}\right] d x\right\| \\
& \quad \leq\left\|\int_{\omega}[\Pi(\nabla y(x))-\nabla y(x)] d x\right\|+\left\|\int_{\omega}\left[\nabla y(x)-F_{\lambda}\right] d x\right\| \\
& \quad \leq(\operatorname{meas} \omega)^{1 / 2}\left[\int_{\omega}\|\Pi(\nabla y(x))-\nabla y(x)\|^{2} d x\right]^{1 / 2}+\left\|\int_{\omega}\left[\nabla y(x)-F_{\lambda}\right] d x\right\| \\
& \quad \leq C\left[\mathcal{E}(y)^{1 / 8}+\mathcal{E}(y)^{1 / 2}\right] .
\end{aligned}
$$

Again by the definition of $\omega_{\rho}^{i}$ and $\omega_{\rho}^{j}$, we get

$$
\begin{aligned}
\left\|\int_{\omega-\left\{\omega_{\rho}^{i} \cup \omega_{\rho}^{j}\right\}} \Pi(\nabla y(x)) d x\right\| \\
\leq C \operatorname{meas}\left(\omega-\left\{\omega_{\rho}^{i} \cup \omega_{\rho}^{j}\right\}\right) \\
\leq \frac{C}{\rho} \int_{\omega-\left\{\omega_{\rho}^{i} \cup \omega_{\rho}^{j}\right\}}\|\Pi(\nabla y(x))-\nabla y(x)\| d x \\
\leq \frac{C(\operatorname{meas} \omega)^{1 / 2}}{\rho}\left[\int_{\omega}\|\Pi(\nabla y(x))-\nabla y(x)\|^{2} d x\right]^{1 / 2} \\
\leq C\left[\mathcal{E}(y)^{1 / 4}+\mathcal{E}(y)^{1 / 2}\right],
\end{aligned}
$$

where the last step follows from Theorem 4.3. 
It now follows from (4.35) and (4.36) that

$$
\begin{aligned}
& \left\|\left[\operatorname{meas} \omega_{\rho}^{i}-\lambda \operatorname{meas} \omega\right] Q U_{i}+\left[\operatorname{meas} \omega_{\rho}^{j}-(1-\lambda) \operatorname{meas} \omega\right] U_{j}\right\| \\
& \quad \leq C\left[\mathcal{E}(y)^{1 / 8}+\mathcal{E}(y)^{1 / 2}\right],
\end{aligned}
$$

which, together with the fact that $Q U_{i}$ and $U_{j}$ are linearly independent, implies the assertion of the theorem.

We now denote by $\mathcal{V}$ the Sobolev space of all measurable functions $f: \Omega \times \mathbb{R}^{3 \times 3} \rightarrow \mathbb{R}$ such that

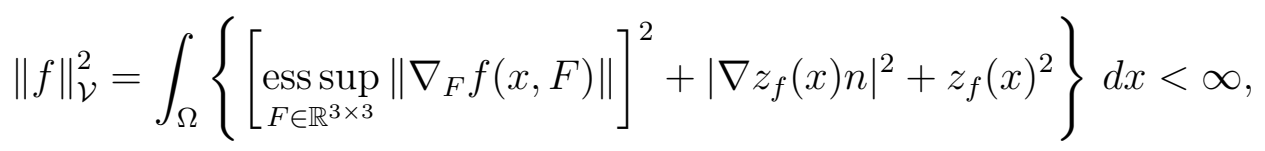

where $z_{f}: \Omega \rightarrow \mathbb{R}$ is defined by

$$
z_{f}(x)=f\left(x, Q U_{i}\right)-f\left(x, U_{j}\right), \quad x \in \Omega .
$$

We can replace the entire gradient space $\mathbb{R}^{3 \times 3}$ in the definition of the norm $\|f\|_{\mathcal{V}}$ by a ball $\left\{F \in \mathbb{R}^{3 \times 3}:\|F\| \leq R\right\}$ for some positive constant $R$ by restricting the gradients of admissible deformations to lie in such a ball. We note that it is not reasonable to use elasticity theory to model deformations with arbitrarily large gradients.

The following theorem gives error bounds for the approximation of nonlinear integrals of deformation gradients which can represent macroscopic thermodynamic densities.

Theorem 4.5. We have

$$
\begin{aligned}
& \mid \int_{\Omega}\{f(x,\left.\nabla y(x))-\left[\lambda f\left(x, Q U_{i}\right)+(1-\lambda) f\left(x, U_{j}\right)\right]\right\} d x \mid \\
& \leq C\|f\|_{\mathcal{V}}\left[\mathcal{E}(y)^{1 / 4}+\mathcal{E}(y)^{1 / 2}\right] \quad \forall f \in \mathcal{V}, \forall y \in \mathcal{A} .
\end{aligned}
$$

Proof. Fix $f \in \mathcal{V}$ and $y \in \mathcal{A}$. We have the decomposition

$$
\begin{aligned}
\int_{\Omega}\{f & \left.(x, \nabla y(x))-\left[\lambda f\left(x, Q U_{i}\right)+(1-\lambda) f\left(x, U_{j}\right)\right]\right\} d x \\
= & \int_{\Omega}[f(x, \nabla y(x))-f(x, \Pi(\nabla y(x)))] d x \\
& \quad+\int_{\Omega}\left\{f(x, \Pi(\nabla y(x)))-\left[\lambda f\left(x, Q U_{i}\right)+(1-\lambda) f\left(x, U_{j}\right)\right]\right\} d x \\
= & J_{2}(f ; y)+J_{3}(f ; y) .
\end{aligned}
$$

It follows from the Cauchy-Schwarz inequality and Theorem 4.3 that

$$
\begin{aligned}
\left|J_{2}(f ; y)\right| & :=\left|\int_{\Omega}[f(x, \nabla y(x))-f(x, \Pi(\nabla y(x)))] d x\right| \\
& \leq \int_{\Omega}\left[\underset{F \in \mathbb{R}^{3 \times 3}}{\operatorname{ess} \sup _{F}}\left\|\nabla_{F} f(x, F)\right\|\right]\|\nabla y(x)-\Pi(\nabla y(x))\| d x
\end{aligned}
$$




$$
\begin{aligned}
& \leq\left\{\int_{\Omega}\left[\underset{F \in \mathbb{R}^{3 \times 3}}{\operatorname{ess} \operatorname{up}_{F}}\left\|\nabla_{F} f(x, F)\right\|\right]^{2} d x\right\}^{1 / 2}\left\{\int_{\Omega}\|\nabla y(x)-\Pi(\nabla y(x))\|^{2} d x\right\}^{1 / 2} \\
& \leq C\|f\|_{\mathcal{V}}\left[\mathcal{E}(y)^{1 / 4}+\mathcal{E}(y)^{1 / 2}\right] .
\end{aligned}
$$

By the rank-one connection (2.7), the definition of $F_{\lambda}(2.10)$, and the definition of the function $z_{f}: \Omega \rightarrow \mathbb{R}$, we have the identity

$$
\begin{aligned}
& f(x, \Pi(F))-\left[\lambda f\left(x, Q U_{i}\right)+(1-\lambda) f\left(x, U_{j}\right)\right] \\
& \quad=\frac{1}{|a|^{2}}\left\{a \cdot\left[\Pi(F)-F_{\lambda}\right] n\right\} z_{f}(x) \quad \forall x \in \Omega, \forall F \in \mathbb{R}^{3 \times 3} .
\end{aligned}
$$

This, together with an integration by parts, leads to

$$
\begin{aligned}
J_{3}(f ; y):= & \int_{\Omega}\left\{f(x, \Pi(\nabla y(x)))-\left[\lambda f\left(x, Q U_{i}\right)+(1-\lambda) f\left(x, U_{j}\right)\right]\right\} d x \\
= & \frac{1}{|a|^{2}} \int_{\Omega}\left\{a \cdot\left[\Pi(\nabla y(x))-F_{\lambda}\right] n\right\} z_{f}(x) d x \\
= & \frac{1}{|a|^{2}} \int_{\Omega}\{a \cdot[\Pi(\nabla y(x))-\nabla y(x)] n\} z_{f}(x) d x \\
& \quad+\frac{1}{|a|^{2}} \int_{\Omega}\left\{a \cdot\left[\nabla y(x)-F_{\lambda}\right] n\right\} z_{f}(x) d x \\
= & \frac{1}{|a|^{2}} \int_{\Omega}\{a \cdot[\Pi(\nabla y(x))-\nabla y(x)] n\} z_{f}(x) d x \\
& \quad-\frac{1}{|a|^{2}} \int_{\Omega}\left\{a \cdot\left[y(x)-F_{\lambda} x\right]\right\}\left[\nabla z_{f}(x) \cdot n\right] d x .
\end{aligned}
$$

Consequently, we have by the Cauchy-Schwarz inequality, Theorem 4.3, and Theorem 4.2 that

$$
\left|J_{3}(f ; y)\right| \leq C\|f\|_{\mathcal{V}}\left[\mathcal{E}(y)^{1 / 4}+\mathcal{E}(y)^{1 / 2}\right] .
$$

Now, the desired inequality follows from (4.37), (4.38), and (4.39).

\section{Finite Element Approximations}

In this section, we continue to assume as in the last section that the continuous energy density $\phi: \mathbb{R}^{3 \times 3} \rightarrow \mathbb{R}$ satisfies (2.3) and (4.1), and that the parameters $\xi, \eta$, and $\zeta$ satisfy the conditions (4.3), (4.4), or (4.5) that can lead to the uniqueness of the Young measure. For simplicity, we also assume that the reference configuration of the crystal $\Omega \subset \mathbb{R}^{3}$ is a polygonal domain so that it can be covered by a finite element mesh consisting of polyhedra. For a treatment of a more general Lipschitz domain, we refer to [32].

We denote by $\left\{\tau_{h}: 0<h \leq h_{0}\right\}$ a family of finite element meshes of $\Omega \subset \mathbb{R}^{3}$, where $h_{0}>0$ is a constant. We assume that each $\tau_{h}$ is a collection of polyhedra $\{K\}$ satisfying $[17,39]$ :

(1) $\bar{\Omega}=\cup_{K \in \tau_{h}} K$; 
(2) interior $K_{1} \cap$ interior $K_{2}=\emptyset$, if $K_{1} \neq K_{2}$ for $K_{1}, K_{2} \in \tau_{h}$;

(3) if $S=K_{1} \cap K_{2} \neq \emptyset$ for $K_{1} \neq K_{2}, K_{1}, K_{2} \in \tau_{h}$, then $S$ is a common face, edge, or vertex of $K_{1}$ and $K_{2}$;

(4) diam $K \leq h$ for all $K \in \tau_{h}$.

For each $h \in\left(0, h_{0}\right]$, we denote by $V_{h}$ a finite element space consisting of piecewise polynomials with respect to the finite element mesh $\tau_{h}$. We assume

(1) $V_{h}$ contains all continuous, piecewise polynomials with respect to $\tau_{h}$. (Hence $V_{h}$ is a conforming finite element space in the sense that $\left.V_{h} \subset W^{1, \infty}\left(\Omega ; \mathbb{R}^{3}\right)\right)$;

(2) there exists an interpolation operator $\mathcal{I}_{h}: W^{1, \infty}(\Omega ; \mathbb{R}) \rightarrow V_{h}$ such that

$$
\operatorname{ess} \sup _{x \in \Omega}\left\|\nabla \mathcal{I}_{h} u(x)\right\| \leq C \operatorname{ess} \sup _{x \in \Omega}\|\nabla u(x)\| \quad \forall u \in W^{1, \infty}(\Omega),
$$

where the constant $C$ above and below will always denote a generic positive constant independent of $h$.

We remark that the most widely used $P_{k}$ or $Q_{k}$ type conforming finite elements, in particular, the $P_{1}$ linear elements defined on tetrahedra and the $Q_{1}$ trilinear elements defined on rectangular parallelepipeds, satisfy these assumptions (for quasi-regular meshes) $[17,39]$.

We now define for each $h \in\left(0, h_{0}\right]$ the set of admissible finite element deformations by

$$
\mathcal{A}_{h}:=\left\{y_{h}=\left(y_{1}, y_{2}, y_{3}\right) \in \mathcal{A}: y_{k} \in V_{h}, k=1,2,3\right\},
$$

where $\mathcal{A}$ is the set of admissible deformations defined in (4.2). Obviously, each $\mathcal{A}_{h} \neq \emptyset$.

The following theorem gives the existence of finite element energy minimizers as well as an error estimate for the corresponding minimum energy.

Theorem 5.1. For each $h \in\left(0, h_{0}\right]$, there exists $y_{h} \in \mathcal{A}_{h}$ such that

$$
\mathcal{E}\left(y_{h}\right)=\min _{z_{h} \in \mathcal{A}_{h}} \mathcal{E}\left(z_{h}\right) \leq C h^{1 / 2} .
$$

Proof. By the Poincaré inequality (cf. [3, 44]) and the quadratic growth rate (4.1) of $\phi: \mathbb{R}^{3 \times 3} \rightarrow \mathbb{R}$, we have

$$
\begin{aligned}
\|y\|_{W^{1,2}\left(\Omega ; \mathbb{R}^{3}\right)} & \leq C\left[\|\nabla y\|_{L^{2}\left(\Omega ; \mathbb{R}^{3 \times 3}\right)}+1\right] \\
& \leq C\left[1+\|\nabla y-\pi(\nabla y)\|_{L^{2}\left(\Omega ; \mathbb{R}^{3 \times 3}\right)}+\|\pi(\nabla y)\|_{L^{2}\left(\Omega ; \mathbb{R}^{3 \times 3}\right)}\right] \\
& \leq C\left[\mathcal{E}(y)^{1 / 2}+1\right] .
\end{aligned}
$$

Since the restriction of $\mathcal{E}$ on the finite-dimensional affine space $\mathcal{A}_{h}$ is continuous, we thus obtain the existence of a finite element energy minimizer $y_{h} \in \mathcal{A}_{h}$ by a usual argument of compactness.

Following $[14,33,34]$, one can construct a finite element deformation $z_{h} \in \mathcal{A}_{h}$ such that

$$
\mathcal{E}\left(z_{h}\right) \leq C h^{1 / 2} .
$$

This finishes the proof. 
Computational results show that number of local minima of the problem

$$
\inf _{v_{h} \in \mathcal{A}_{h}} \mathcal{E}\left(v_{h}\right)
$$

grows arbitrarily large as the mesh size $h \rightarrow 0$ [34]. Many of these local minima are approximations on different length scales to the same optimal microstructure [34]. Thus, it is reasonable to give error estimates for finite element approximations $y_{h} \in \mathcal{A}_{h}$ satisfying the quasi-optimality condition

$$
\mathcal{E}\left(y_{h}\right) \leq \sigma \inf _{z_{h} \in \mathcal{A}_{h}} \mathcal{E}\left(z_{h}\right)
$$

for some constant $\sigma \geq 1$ independent of $h$.

It follows directly from the above theorem and our stability theory established in $\S 4$ (cf. Theorem 4.2, Theorem 4.3, Theorem 4.4, and Theorem 4.5) that we can obtain the following error estimates for finite element quasi-minimizers $y_{h} \in \mathcal{A}_{h}$ defined by (5.1).

Corollary 5.1. (1) We have

$$
\int_{\Omega}\left|y_{h}(x)-F_{\lambda} x\right|^{2} d x \leq C h^{1 / 4}
$$

and

$$
\int_{\Omega}\left\|\nabla y_{h}(x)-\Pi\left(\nabla y_{h}(x)\right)\right\|^{2} d x \leq C h^{1 / 4}
$$

for any $y_{h} \in \mathcal{A}_{h}$ satisfying (5.1).

(2) For any $w \in \mathbb{R}^{3}$ such that $w \cdot n=0$ and $|w|=1$, we have

$$
\int_{\Omega}\left|\left[\nabla y_{h}(x)-F_{\lambda}\right] w\right|^{2} d x \leq C h^{1 / 4}
$$

for any $y_{h} \in \mathcal{A}_{h}$ satisfying (5.1). that

(3) If $\omega \subset \Omega$ is a Lipschitz domain, then there exists a constant $C=C(\omega)>0$ such

$$
\left\|\int_{\omega}\left[\nabla y_{h}(x)-F_{\lambda}\right] d x\right\| \leq C h^{1 / 16}
$$

for any $y_{h} \in \mathcal{A}_{h}$ satisfying (5.1).

Corollary 5.2. (1) If $\omega \subset \Omega$ is a Lipschitz domain and $\rho>0$, then there exists a constant $C=C(\omega, \rho)>0$ such that

$$
\left|\frac{\operatorname{meas} \omega_{\rho}^{i}\left(y_{h}\right)}{\operatorname{meas} \omega}-\lambda\right|+\left|\frac{\operatorname{meas} \omega_{\rho}^{j}\left(y_{h}\right)}{\operatorname{meas} \omega}-(1-\lambda)\right| \leq C h^{1 / 16}
$$

for any $y_{h} \in \mathcal{A}_{h}$ satisfying (5.1), where for $y \in \mathcal{A}$ the sets $\omega_{\rho}^{i}(y)$ and $\omega_{\rho}^{j}(y)$ are defined in $\S 4.3$.

(2) We have

$$
\left|\int_{\Omega}\left\{f\left(x, \nabla y_{h}(x)\right)-\left[\lambda f\left(x, Q U_{i}\right)+(1-\lambda) f\left(x, U_{j}\right)\right]\right\} d x\right| \leq C\|f\|_{\mathcal{V}} h^{1 / 8}
$$


for any $f \in \mathcal{V}$ and any $y_{h} \in \mathcal{A}_{h}$ satisfying (5.1), where the space $\mathcal{V}$ is defined in $\S 4.3$.

\section{REFERENCES}

[1] Rohan Abeyaratne, Chun-Hwa Chu, and Richard D. James. Kinetics and hysteresis in martensitic single crystals. In Proc. Symposium on the Mechanics of Phase Transformations and Shape Memory Alloys. ASME, 1994.

[2] Rohan Abeyaratne, Chun-Hwa Chu, and Richard D. James. Kinetics of materials with wiggly energies: theory and application to the evolution of twinnig microstructures in a $\mathrm{Cu}-\mathrm{Al}-\mathrm{Ni}$ shape memory alloy. Phil. Mag. A, 73(2):457-497, 1996.

[3] Robert A. Adams. Sobolev Spaces. Academic Press, New York, 1975.

[4] John M. Ball. Constitutive inequalities and existence theorems in nonlinear elastostatics. In R. Knops, editor, Nonlinear Analysis and Mechanics: Heriot-Watt Symposium I, volume 17 of Research Notes in Mathematics, pages 187-241. Pitman, 1977.

[5] John M. Ball. Convexity conditions and existence theorems in nonlinear elasticity. Arch. Rat. Mech. Anal., 63:337-403, 1977.

[6] John M. Ball. A version of the fundamental theorem for Young measures. In Michel Rascle, Denis Serre, and Marshall Slemrod, editors, PDEs and Continuum Models of Phase Transition, pages 207-215, 1989.

[7] John M. Ball, Chun-Hwa Chu, and Richard D. James. Hysteresis during stress-induced variant rearrangement. In Proceedings of the International Conference on Martensitic Transformations, 1995.

[8] John M. Ball, J. C. Currie, and Peter J. Olver. Null Lagrangians, weak continuity and variational problems of arbitrary order. J. Funct. Anal., 41:135-174, 1981.

[9] John M. Ball and Richard D. James. Fine phase mixtures as minimizers of energy. Arch. Rat. Mech. Anal., 100:13-52, 1987.

[10] John M. Ball and Richard D. James. Proposed experimental tests of a theory of fine microstructure and the two-well problem. Phil. Trans. R. Soc. Lond. A, 338:389-450, 1992.

[11] Kaushik Bhattacharya. Wedge-like microstructure in martensite. Acta Metall. Mater., 39:2431$2444,1991$.

[12] Kaushik Bhattacharya. Self accomodation in martensite. Arch. Rat. Mech. Anal., 120:201-244, 1992.

[13] Kaushik Bhattacharya. Theory of martensitic microstructure and the shape-memory effect. In I. Müller G. Airoldi and S. Miyazaki, editors, Shape-memory alloys: from microstructure to macroscopic properties. Trans Tech Publications, 1998.

[14] Michel Chipot, Charles Collins, and David Kinderlehrer. Numerical analysis of oscillations in multiple well problems. Numer. Math., 70:259-282, 1995.

[15] Michel Chipot and David Kinderlehrer. Equilibrium configurations of crystals. Arch. Rat. Mech. Anal., 103:237-277, 1988.

[16] Chun-Hwa Chu. Hysteresis and microstructures: a study of biaxial loading on compound twins of Copper-Aluminum-Nickel single crystals. PhD thesis, University of Minnesota, 1993.

[17] Philippe G. Ciarlet. The Finite Element Method for Elliptic Problems. North-Holland, Amsterdam, 1978.

[18] Bernard Dacorogna. Direct methods in the calculus of variations. Springer-Verlag, Berlin, 1989.

[19] Jerald Ericksen. Nilpotent energies in liquid crystal theory. Arch. Rat. Mech. Anal., 10:951-957, 1962.

[20] Jerald Ericksen. Constitutive theory for some constrained elastic crystals. Int. J. Solids and Structures, 22:951-964, 1986.

[21] Jerald Ericksen. Stable equilibrium configurations of elastic crystals. Arch. Rat. Mech. Anal., 94:1-14, 1986. 
[22] J. C. Escobar and R. J. Clifton. On pressure shear plate impact for studying the kinetics of stress-induced phase-transformations. Mat. Sci. Engng. A, 170:125-142, 1993.

[23] Hiroyasu Funakubo, editor. Shape memory alloys. Gordon and Breach Science Publishers, New York, 1987.

[24] Richard D. James and David Kinderlehrer. Theory of diffusionless phase transitions. In Michel Rascle, Denis Serre, and Marshall Slemrod, editors, PDEs and continuum models of phase transitions, volume 344 of Lecture Notes in Physics, pages 51-84. Springer-Verlag, 1989.

[25] Richard D. James, Robert V. Kohn, and Thomas W. Shield. Modeling the branched needle microstructures at the edge of a martensite laminate. J. de Physique IV, 5:253-259, 1995. ICOMAT95.

[26] David Kinderlehrer and Pablo Pedregal. Characterizations of gradient Young measures. Arch. Rat. Mech. Anal., 115:329-365, 1991.

[27] Robert V. Kohn. Relaxation of a double-well energy. Cont. Mech. Thermodyn., 3:193-236, 1991.

[28] Bo Li. Analysis and computation of martensitic microstructure. PhD thesis, University of Minnesota, 1996.

[29] Bo Li and Mitchell Luskin. Numerical simulation of needle-like microstructure in martensitic crystals. Manuscript, 1996.

[30] Bo Li and Mitchell Luskin. Finite element analysis of microstructure for the cubic to tetragonal transformation. SIAM J. Numer. Anal., 35(1):376-392, 1998.

[31] Bo Li and Mitchell Luskin. Nonconforming finite element approximation of crystalline microstructure. Math. Comp., 67:917-946, 1998.

[32] Bo Li and Mitchell Luskin. Approximation of a martensitic laminate with varying volume fractions. Math. Model. Numer. Anal., 1998 (to appear).

[33] Mitchell Luskin. Approximation of a laminated microstructure for a rotationally invariant, double well energy density. Numer. Math., 75:205-221, 1996.

[34] Mitchell Luskin. On the computation of crystalline microstructure. Acta Numerica, pages 191$257,1996$.

[35] Mitchell Luskin and Ling Ma. Analysis of the finite element approximation of microstructure in micromagnetics. SIAM J. Numer. Anal., 29:320-331, 1992.

[36] Charles Bradfield Morrey, Jr. Multiple Integrals in the Calculus of Variations. Springer, Berlin, Heidelberg, New York, 1966.

[37] Stefan Müller. A sharp version of Zhang's theorem on truncating sequences of gradients. Trans. $A M S, 1998$ (to appear).

[38] K. Otsuka and K. Shimizu. Morphology and crystallography of thermoelastic Cu-Al-Ni martensite analyzed by the phenomenological theory. Trans. JIM, 15:103-108, 1974.

[39] Alfio Quarteroni and Alberto Valli. Numerical Approximation of Partial Differential Equations. Springer-Verlag, Berlin, 1994.

[40] Y. G. Reshetnyak. On the stability of conformal mappings in multi-dimensional spaces. Siberian Math. J., 8:69-85, 1967.

[41] Vladimir Sverák. Lower-semicontinuity of variational integrals and compensated compactness. In Proceedings ICM 94, Zürich, 1995. Birkhäuser.

[42] S. S. Tan and H. B. Xu. Observations on a CuAlNi single crystal. Cont. Mech. Thermodyn., 2:241-244, 1990.

[43] Luc Tartar. Compensated compactness and applications to partial differential equations. In R. Knops, editor, Nonlinear analysis and mechanics, volume 39 of Pitman Research Notes in Mathematics, pages 136-212, London, 1978. Pitman.

[44] Joseph Wloka. Partial Differential Equations. Cambridge University Press, Cambridge, 1987.

[45] Kewei Zhang. A construction of quasiconvex functions with linear growth at infinity. Ann. Scuola Norm. Sup. Pisa, 19:313-326, 1992. 
Division of Engineering and Applied Science, Mail Code 104-44, California Institute of Technology, Pasadena, CA 91125, U.S.A.

E-mail address: bhatta@cco.caltech.edu

Department of Mathematics, University of Maryland, College Park, MD 20742, U.S.A.

E-mail address: bli@math.umd.edu

School of Mathematics, University of Minnesota, 206 Church Street, S.E., MinNEAPOLIS, MN 55455, U.S.A.

E-mail address: luskin@math.umn.edu 